\begin{tabular}{|c|l|}
\hline Title & Rotational motion of traveling spots in dissipative systems \\
\hline Author(s) & Teramoto, Takashi; Suzuki, Katsuya; Nishiura, Y asumasa \\
\hline Citation & $\begin{array}{l}\text { Physical Review E, 80(4), 046208 } \\
\text { https://doi.org/40.1103/PhysRevE.80.046208 }\end{array}$ \\
\hline Issue Date & 2009-10 \\
\hline Doc URL & http://hdl.handle.net/2115/39847 \\
\hline Rights & ○ 2009 The A American Physical Society \\
\hline Type & article \\
\hline File Information & PRE80-4_046208.pdf \\
\hline
\end{tabular}

Instructions for use 


\title{
Rotational motion of traveling spots in dissipative systems
}

\author{
Takashi Teramoto \\ Faculty of Photonics Science, Chitose Institute of Science and Technology, Chitose 066-8655, Japan
}

Katsuya Suzuki and Yasumasa Nishiura

Research Institute for Electronic Science, Hokkaido University, Sapporo 001-0020, Japan

(Received 2 April 2009; revised manuscript received 30 June 2009; published 20 October 2009)

\begin{abstract}
What is the origin of rotational motion? An answer is presented through the study of the dynamics for spatially localized spots near codimension 2 singularity consisting of drift and peanut instabilities. The drift instability causes a head-tail asymmetry in spot shape, and the peanut one implies a deformation from circular to peanut shape. Rotational motion of spots can be produced by combining these instabilities in a class of three-component reaction-diffusion systems. Partial differential equations dynamics can be reduced to a finitedimensional one by projecting it to slow modes. Such a reduction clarifies the bifurcational origin of rotational motion of traveling spots in two dimensions in close analogy to the normal form of 1:2 mode interactions.
\end{abstract}

DOI: 10.1103/PhysRevE.80.046208

PACS number(s): 82.40.Bj, 05.45.-a, 82.40.Ck

Spatially localized moving objects such as traveling pulses and self-propelled particles are fundamental objects arising in nonlinear science, which display a large variety of dynamical behaviors in many dissipative systems [1-4]. In two dimensions, traveling motion causes symmetry breaking from the circular shape of a standing spot. The onset of a straight motion of traveling spots has been studied for a drift instability $[3,5,6]$, in which the authors showed a drift pitchfork scenario from the local properties at a codimension 1 bifurcation. This topic has attracted much interest experimentally as well as theoretically with respect to driven droplet patterns on a solid substrate [7-9]. In biological tissues, digital image analysis also shows that a head-tail asymmetry in cell shape determines the direction of motion and some sorts of interference wave pattern occurs during spontaneous cell migration $[10,11]$. These recent experiments allow us to deduce the underlying mechanism of interplay between the spot locomotion and shape-change dynamics.

In this paper, we consider the spot dynamics near a codimension 2 singularity for reaction-diffusion systems in which the associated parameter values are located close to the drift and peanut bifurcation points. Drift instability originates in the translation-free mode and the associated deformation eigenvector represents a $\mathcal{D}_{1}$ symmetry breaking from a disk shape. Peanut one is by $\mathcal{D}_{2}$ symmetry-breaking bifurcation, corresponding to two-mode deformation, where $\mathcal{D}_{n}$ stands for the dihedral symmetry group. We show that such a codimension 2 singularity can induce rotational motion of traveling spots - that is, rotational spot (RS) motion-in a class of reaction-diffusion systems.

The occurrence of such a motion is generic because the original partial differential equations (PDEs) can be reduced to finite-dimensional ordinary differential equations (ODEs) based on the center manifold theory [12-14], and the resulting ODEs take a normal form of 1:2 mode interaction of cubic type. We analyze the reduced ODEs, and show that there exists a solution in which both drift velocity vector and peanut deformation become time-periodic functions that correspond to the rotational motion of traveling spot solution to the original reaction-diffusion systems. The information specific to the form and parameters of the original PDEs is con- tained in the coefficients of the reduced system. We also discuss about the relationship between the global bifurcational structures of the original PDEs and the reduced ODEs, which sheds light on the origin of rotational motion, that is, such a motion emerges through the interaction between drift and peanut instabilities and it is realized in the PDE counterpart, i.e., the three-component reaction-diffusion system.

A general setup for the PDE system in a neighborhood of codimension 2 bifurcation point $\boldsymbol{\lambda}^{c}=\left(\lambda_{1}^{c}, \lambda_{2}^{c}\right)$ reads, with a small parameter $\boldsymbol{\eta}=\left(\eta_{1}, \eta_{2}\right)$ as $\boldsymbol{\lambda}=\boldsymbol{\lambda}^{c}+\boldsymbol{\eta}$,

$$
\boldsymbol{u}_{t}=D \Delta \boldsymbol{u}+F(\boldsymbol{u} ; \boldsymbol{\lambda}) \equiv \mathcal{L}\left(\boldsymbol{u} ; \boldsymbol{\lambda}^{c}\right)+\sum_{i=1}^{2} \eta_{i} \boldsymbol{g}_{i}(\boldsymbol{u}),
$$

where $\boldsymbol{g}_{i}(i=1,2)$ is $N$-dimensional vector-valued functions. Let $X:=\left\{L^{2}(\mathbb{R})\right\}^{N}, \quad \boldsymbol{u}(t, \boldsymbol{r})=\left(u_{1}, \cdots, u_{N}\right)^{T} \in X, \quad$ be $\quad$ an $N$-dimensional vector and $F: \mathbb{R}^{N} \rightarrow \mathbb{R}^{N}, D$ be a positive diagonal matrix. We assume that the nontrivial standing spot solution $S(\boldsymbol{r} ; \boldsymbol{\lambda})$ exists at $\boldsymbol{\lambda}=\boldsymbol{\lambda}^{c}$, i.e., $\mathcal{L}\left(S ; \boldsymbol{\lambda}^{c}\right)=0$.

Let $L$ be the linearized operator $L=\mathcal{L}^{\prime}\left[S\left(\boldsymbol{r} ; \boldsymbol{\lambda}^{c}\right)\right] . L$ has a codimension 2 singularity at $\boldsymbol{\lambda}=\boldsymbol{\lambda}^{c}$ consisting of drift and peanut bifurcations in addition to the translation-free 0 eigenvalue; that is, there exist three types of eigenfunctions $\boldsymbol{\phi}_{i}(\boldsymbol{r}), \boldsymbol{\psi}_{i}(\boldsymbol{r})$, and $\boldsymbol{\xi}_{i}(\boldsymbol{r})(i=1,2)$ such that $L \boldsymbol{\phi}_{i}=0, L \boldsymbol{\psi}_{i}=-\boldsymbol{\phi}_{i}$, and $L \boldsymbol{\xi}_{i}=0$, where $\boldsymbol{\phi}_{i}=\partial S / \partial x_{i}$ and $\boldsymbol{\psi}_{i}$ represents the deformation vector with Jordan form for the drift bifurcation. $\boldsymbol{\xi}_{i}^{*}$ is the $\mathcal{D}_{2}$-symmetry breaking eigenfunction of peanut shape.

Similar properties also hold for $L^{*}$, that is, there exist $\boldsymbol{\phi}_{i}^{*}$, $\boldsymbol{\psi}_{i}^{*}$, and $\boldsymbol{\xi}_{i}^{*}$ such that $L^{*} \boldsymbol{\phi}_{i}^{*}=0, L^{*} \boldsymbol{\psi}_{i}^{*}=-\boldsymbol{\phi}_{i}^{*}$, and $L^{*} \xi_{i}^{*}=0$. Let $E=\operatorname{span}\left\{\boldsymbol{\phi}_{i}, \boldsymbol{\psi}_{i}, \boldsymbol{\xi}_{i}\right\}$ and the eigenfunctions be normalized by $\left\langle\boldsymbol{\psi}_{i}, \boldsymbol{\phi}_{j}\right\rangle_{L^{2}}=\left\langle\boldsymbol{\psi}_{i}, \boldsymbol{\psi}_{j}^{*}\right\rangle_{L^{2}}=0$, and

$$
\left\langle\boldsymbol{\phi}_{i}, \boldsymbol{\psi}_{j}^{*}\right\rangle_{L^{2}}=\left\langle\boldsymbol{\psi}_{i}, \boldsymbol{\phi}_{j}^{*}\right\rangle_{L^{2}}=\left\langle\boldsymbol{\xi}_{i}, \boldsymbol{\xi}_{j}^{*}\right\rangle_{L^{2}}=\left\{\begin{array}{rr}
\pi, & i=j \\
0, & i \neq j .
\end{array}\right.
$$

The motion of a spot solution $\boldsymbol{u}$ is essentially described by the two-dimensional vector functions of time $t ; \boldsymbol{p}=\left(p_{1}, p_{2}\right)$ denotes the location of the spot; $\boldsymbol{q}=\left(q_{1}, q_{2}\right)$ denotes its velocity; and $\boldsymbol{s}=\left(s_{1}, s_{2}\right)$ denotes its deformation. For small $\boldsymbol{\eta}$, we can approximate a solution $\boldsymbol{u}$ by 


$$
\boldsymbol{U}=\tau(\boldsymbol{p})\left\{S(\boldsymbol{r})+\sum_{i=1}^{2} q_{i} \boldsymbol{\psi}_{i}(\boldsymbol{r})+\sum_{i=1}^{2} s_{i} \boldsymbol{\xi}_{i}(\boldsymbol{r})+\boldsymbol{\zeta}^{\dagger}\right\},
$$

where $\tau(\boldsymbol{p})$ is the translation operator with $[\tau(\boldsymbol{p}) \boldsymbol{u}](\boldsymbol{r})$ $=\boldsymbol{u}(\boldsymbol{r}-\boldsymbol{p})$. The remaining term $\boldsymbol{\zeta}^{\dagger}$ belongs to $E^{\perp}$. More precisely, $\quad \boldsymbol{\zeta}^{\dagger}=q_{1}^{2} \zeta_{1}+q_{2}^{2} \boldsymbol{\zeta}_{2}+q_{1} q_{2} \zeta_{3}+s_{1}^{2} \zeta_{4}+s_{2}^{2} \boldsymbol{\zeta}_{5}+s_{1} s_{2} \zeta_{6}+q_{1} s_{1} \zeta_{7}$ $+q_{2} s_{2} \zeta_{8}+q_{1} s_{2} \zeta_{9}+q_{2} s_{1} \zeta_{10}+\eta_{1} \zeta_{11}+\eta_{2} \zeta_{12}$ with $\zeta_{k}(k=1, \cdots 12)$ $\in E^{\perp}$ are defined by solutions of

$$
\begin{gathered}
L \zeta_{1}+\frac{1}{2} F^{\prime \prime}(S) \boldsymbol{\psi}_{1}^{2}+\boldsymbol{\psi}_{1 x_{1}}=\alpha \boldsymbol{\xi}_{1}, \\
L \boldsymbol{\zeta}_{2}+\frac{1}{2} F^{\prime \prime}(S) \boldsymbol{\psi}_{2}^{2}+\boldsymbol{\psi}_{2 x_{2}}=-\alpha \boldsymbol{\xi}_{1}, \\
L \boldsymbol{\zeta}_{3}+F^{\prime \prime}(S) \boldsymbol{\psi}_{1} \boldsymbol{\psi}_{2}+\boldsymbol{\psi}_{1 x_{2}}+\boldsymbol{\psi}_{2 x_{1}}=2 \alpha \boldsymbol{\xi}_{2},
\end{gathered}
$$

and

$$
\begin{aligned}
& L \boldsymbol{\zeta}_{4}+\frac{1}{2} F^{\prime \prime}(S) \xi_{1}^{2}=0, \\
& L \boldsymbol{\zeta}_{5}+\frac{1}{2} F^{\prime \prime}(S) \xi_{2}^{2}=0, \\
& L \boldsymbol{\zeta}_{6}+F^{\prime \prime}(S) \boldsymbol{\xi}_{1} \xi_{2}=0,
\end{aligned}
$$

and

$$
\begin{gathered}
L \boldsymbol{\zeta}_{7}+F^{\prime \prime}(S) \boldsymbol{\psi}_{1} \xi_{1}+\boldsymbol{\xi}_{1 x_{1}}=\beta \boldsymbol{\psi}_{1}+\beta^{\prime} \boldsymbol{\phi}_{1}, \\
L \boldsymbol{\zeta}_{8}+F^{\prime \prime}(S) \boldsymbol{\psi}_{2} \boldsymbol{\xi}_{2}+\boldsymbol{\xi}_{2 x_{2}}=\beta \boldsymbol{\psi}_{1}+\beta^{\prime} \boldsymbol{\phi}_{1}, \\
L \boldsymbol{\zeta}_{9}+F^{\prime \prime}(S) \boldsymbol{\psi}_{1} \boldsymbol{\xi}_{2}+\boldsymbol{\xi}_{2 x_{1}}=\beta \boldsymbol{\psi}_{2}+\beta^{\prime} \boldsymbol{\phi}_{2}, \\
L \boldsymbol{\zeta}_{10}+F^{\prime \prime}(S) \boldsymbol{\psi}_{2} \boldsymbol{\xi}_{1}+\boldsymbol{\xi}_{1 x_{2}}=-\beta \boldsymbol{\psi}_{2}-\beta^{\prime} \boldsymbol{\phi}_{2},
\end{gathered}
$$

and

$$
\begin{gathered}
L \boldsymbol{\zeta}_{11}+\boldsymbol{g}_{1}(S)=0, \\
L \boldsymbol{\zeta}_{12}+\boldsymbol{g}_{2}(S)=0,
\end{gathered}
$$

where $\alpha, \beta$, and $\beta^{\prime}$ are constants satisfying the following conditions:

$$
\begin{aligned}
& \left\langle F^{\prime \prime}(S) \boldsymbol{\psi}_{1} \boldsymbol{\psi}_{2}+\boldsymbol{\psi}_{1 x_{2}}+\boldsymbol{\psi}_{2 x_{1}}-2 \alpha \boldsymbol{\xi}_{2}, \boldsymbol{\xi}_{2}^{*}\right\rangle_{L^{2}}=0, \\
& \left\langle F^{\prime \prime}(S) \boldsymbol{\psi}_{1} \boldsymbol{\xi}_{2}+\boldsymbol{\xi}_{2 x_{1}}-\beta \boldsymbol{\psi}_{2}-\beta^{\prime} \boldsymbol{\phi}_{2}, \boldsymbol{\phi}_{2}^{*}\right\rangle_{L^{2}}=0, \\
& \left\langle F^{\prime \prime}(S) \boldsymbol{\psi}_{1} \boldsymbol{\xi}_{2}+\boldsymbol{\xi}_{2 x_{1}}-\beta \boldsymbol{\psi}_{2}-\beta^{\prime} \boldsymbol{\phi}_{2}, \boldsymbol{\psi}_{2}^{*}\right\rangle_{L^{2}}=0 .
\end{aligned}
$$

Substituting Eq. (3) into Eq. (1) and taking the inner product with the adjoint eigenfunctions, the principal part of the reduced ODEs for $\left(p_{i}, q_{i}, s_{i}\right)$ is given by

$$
\begin{gathered}
\dot{z}_{0}=z_{1}-\beta^{\prime} \bar{z}_{1} z_{2}, \\
\dot{z}_{1}=M_{1}\left|z_{1}\right|^{2} z_{1}+M_{2}\left|z_{2}\right|^{2} z_{1}+M_{3} z_{1}+\beta \bar{z}_{1} z_{2},
\end{gathered}
$$

$$
\dot{z}_{2}=N_{1}\left|z_{2}\right|^{2} z_{2}+N_{2}\left|z_{1}\right|^{2} z_{2}+N_{3} z_{2}+\alpha z_{1}^{2} .
$$

Here we introduce the complex variables $z_{0}=p_{1}+i p_{2}$, $z_{1}=q_{1}+i q_{2}$, and $z_{2}=s_{1}+i s_{2}$. Note that $\zeta^{\dagger}$ with the conditions of Eqs. (4)-(7) is necessary for computations of cubic terms in Eq. (9).

The constants $M_{1}, M_{2}$, and $M_{3}$ are obtained from the model system (1) as follows:

$$
\begin{aligned}
\pi M_{1}= & \frac{1}{6}\left\langle F^{\prime \prime \prime}(S) \boldsymbol{\psi}_{1}^{3}, \boldsymbol{\phi}_{1}^{*}\right\rangle_{L^{2}}+\left\langle F^{\prime \prime}(S) \boldsymbol{\psi}_{1} \zeta_{1}, \boldsymbol{\phi}_{1}^{*}\right\rangle_{L^{2}}+\left\langle\boldsymbol{\zeta}_{1 x_{1}}, \boldsymbol{\phi}_{1}^{*}\right\rangle_{L^{2}}, \\
\pi M_{2}= & \frac{1}{2}\left\langle F^{\prime \prime \prime}(S) \xi_{1}^{2} \boldsymbol{\psi}_{1}, \boldsymbol{\phi}_{1}^{*}\right\rangle_{L^{2}}+\left\langle F^{\prime \prime}(S) \boldsymbol{\psi}_{1} \boldsymbol{\zeta}_{4}, \boldsymbol{\phi}_{1}^{*}\right\rangle_{L^{2}} \\
& +\left\langle F^{\prime \prime}(S) \boldsymbol{\xi}_{1} \boldsymbol{\zeta}_{7}, \boldsymbol{\phi}_{1}^{*}\right\rangle_{L^{2}}+\left\langle\boldsymbol{\zeta}_{4 x_{1}}, \boldsymbol{\phi}_{1}^{*}\right\rangle_{L^{2}}-\beta^{\prime}\left\langle\boldsymbol{\xi}_{1 x_{1}}, \boldsymbol{\phi}_{1}^{*}\right\rangle_{L^{2}}, \\
\pi M_{3}= & \eta_{1}\left(\left\langle F^{\prime \prime}(S) \boldsymbol{\psi}_{1} \boldsymbol{\zeta}_{11}, \boldsymbol{\phi}_{1}^{*}\right\rangle_{L^{2}}+\left\langle\boldsymbol{g}_{1}^{\prime}(S) \boldsymbol{\psi}_{1}, \boldsymbol{\phi}_{1}^{*}\right\rangle_{L^{2}}\right. \\
& \left.+\left\langle\boldsymbol{\zeta}_{11 x_{1}}, \boldsymbol{\phi}_{1}^{*}\right\rangle_{L^{2}}\right)+\eta_{2}\left(\left\langle F^{\prime \prime}(S) \boldsymbol{\psi}_{1} \zeta_{12}, \boldsymbol{\phi}_{1}^{*}\right\rangle_{L^{2}}\right. \\
& \left.+\left\langle\boldsymbol{g}_{2}^{\prime}(S) \boldsymbol{\psi}_{1}, \boldsymbol{\phi}_{1}^{*}\right\rangle_{L^{2}}+\left\langle\boldsymbol{\zeta}_{12 x_{1}}, \boldsymbol{\phi}_{1}^{*}\right\rangle_{L^{2}}\right) .
\end{aligned}
$$

The constants $N_{1}, N_{2}$, and $N_{3}$ are also obtained as follows:

$$
\pi N_{1}=\frac{1}{6}\left\langle F^{\prime \prime \prime}(S) \boldsymbol{\xi}_{1}^{3}, \boldsymbol{\xi}_{1}^{*}\right\rangle_{L^{2}}+\left\langle F^{\prime \prime}(S) \boldsymbol{\xi}_{1} \boldsymbol{\zeta}_{4}, \boldsymbol{\xi}_{1}^{*}\right\rangle_{L^{2}}
$$

$$
\begin{aligned}
\pi N_{2}= & \frac{1}{2}\left\langle F^{\prime \prime \prime}(S) \boldsymbol{\psi}_{1}^{2} \boldsymbol{\xi}_{1}, \boldsymbol{\xi}_{1}^{*}\right\rangle_{L^{2}}+\left\langle F^{\prime \prime}(S) \boldsymbol{\psi}_{1} \boldsymbol{\zeta}_{7}, \boldsymbol{\xi}_{1}^{*}\right\rangle_{L^{2}} \\
& +\left\langle F^{\prime \prime}(S) \boldsymbol{\xi}_{1} \boldsymbol{\zeta}_{1}, \boldsymbol{\xi}_{1}^{*}\right\rangle_{L^{2}}+\left\langle\boldsymbol{\zeta}_{7 x_{1}}, \boldsymbol{\xi}_{1}^{*}\right\rangle_{L^{2}}-\beta^{\prime}\left\langle\boldsymbol{\psi}_{1 x_{1}}, \boldsymbol{\xi}_{1}^{*}\right\rangle_{L^{2}} \\
\pi N_{3}= & \eta_{1}\left(\left\langle F^{\prime \prime}(S) \boldsymbol{\xi}_{1} \boldsymbol{\zeta}_{11}, \boldsymbol{\xi}_{1}^{*}\right\rangle_{L^{2}}+\left\langle\boldsymbol{g}_{1}^{\prime}(S) \boldsymbol{\xi}_{1}, \boldsymbol{\xi}_{1}^{*}\right\rangle_{L^{2}}\right) \\
& +\eta_{2}\left(\left\langle F^{\prime \prime}(S) \boldsymbol{\xi}_{1} \boldsymbol{\zeta}_{12}, \boldsymbol{\xi}_{1}^{*}\right\rangle_{L^{2}}+\left\langle\boldsymbol{g}_{2}^{\prime}(S) \boldsymbol{\xi}_{1}, \boldsymbol{\xi}_{1}^{*}\right\rangle_{L^{2}}\right)
\end{aligned}
$$

The bifurcation properties are determined by the coefficients of Eqs. (10) and (11), especially their signs, to the reduced systems. The derivations are shown in Appendixes A and B.

The dynamics of Eq. (9) are essentially governed by the last two equations, exactly the same as the normal form obtained in the study of resonance patterns in a bilayer fluid under $O(2)$-symmetry operations $[15,16]$. It is natural that the relationship between drift and peanut deformations viewed from a circular shape is analogous to the 1:2 mode interactions. Letting $z_{1}=Q e^{i \phi}$ and $z_{2}=S e^{i \psi}$, we rewrite Eq. (9) as

$$
\begin{gathered}
\dot{Q}=\left(M_{1} Q^{2}+M_{2} S^{2}+M_{3}\right) Q+\beta Q S \cos \theta, \\
\dot{S}=\left(N_{1} S^{2}+N_{2} Q^{2}+N_{3}\right) S+\alpha Q^{2} \cos \theta, \\
\dot{\theta}=-\left(2 \beta S+\frac{\alpha Q^{2}}{S}\right) \sin \theta,
\end{gathered}
$$

where we set $\theta=\psi-2 \phi$. In addition to the trivial standing disk (SD) spot of $Q=S=0$, we have the fixed points of Eq. (12) with $|\cos \theta|=1$ as the standing peanut (SP) spot of $Q=0$ and $S^{2}=-N_{3} / N_{1}$. Hereafter we use $\left(M_{3}, N_{3}\right)$ as the new bifurcation parameter set. 
The traveling spot solution of Eq. (13) bifurcates from the SD spot at $M_{3}=0$ and from the SP spot at $M_{3}-M_{2} N_{3} / N_{1} \pm \beta\left(-N_{3} / N_{1}\right)^{1 / 2}=0$,

$$
\begin{gathered}
M_{1} Q^{2}+M_{2} S^{2}+M_{3} \pm \beta S=0, \\
\left(N_{1} S^{2}+N_{2} Q^{2}+N_{3}\right) S \pm \alpha Q^{2}=0,
\end{gathered}
$$

where the traveling spot $\mathrm{TS}_{0}$ with $\cos \theta=1 \quad\left(\mathrm{TS}_{\pi}\right.$ with $\cos \theta=-1)$ corresponds to a propagation direction parallel (perpendicular) to the long axis of the deformed shape. The stability and its properties of traveling spot solutions can be obtained by investigating the linearized system of Eq. (9).

As a representative model system fitting our framework, we employ the following activator-substrate-inhibitor reaction-diffusion system:

$$
\begin{gathered}
u_{t}=D_{u} \Delta u-\frac{u v^{2}}{1+f_{2} w}+f_{0}(1-u), \\
v_{t}=D_{v} \Delta v+\frac{u v^{2}}{1+f_{2} w}-\left(f_{0}+f_{1}\right) v, \\
\tau w_{t}=D_{w} \Delta w+f_{3}(v-w),
\end{gathered}
$$

where we set the parameter values $f_{0}=0.05, f_{2}=0.5, f_{3}=0.2$, $\left(D_{u}, D_{v}, D_{w}\right)=\left(2.0 \times 10^{-4}, 1.0 \times 10^{-4}, 5.0 \times 10^{-4}\right)$, and $\tau=40$ [4]. As shown in Fig. 1(a), by numerical analysis of Eq. (14), we found that the drift and peanut bifurcations occur on the SD branch and the profiles of the associated eigenfunctions are shown in Fig. 1(c). The SP branch appears subcritically at $f_{1} \approx 0.0592$ and traveling spot solutions of $\mathrm{TS}_{\pi}$ and $\mathrm{TS}_{0}$ emanate from the drift bifurcation points at $f_{1} \approx 0.0605$ and 0.0617 on the SP branch, respectively. Especially, the solution profile of $\mathrm{TS}_{\pi}$ is deformed from peanut shape to disk shape via saddle-node bifurcation. Its stability property changes from saddle to unstable spiral just after the saddlenode point at $f_{1} \approx 0.06027$. As shown in Figs. 1(a) and 1(b), $\mathrm{TS}_{\pi}$ recovers its stability via Hopf bifurcation at $f_{1} \approx 0.06034$ and end up with the drift bifurcation point at $f_{1} \approx 0.0624$ on the SD branch. We also detect pitchfork bifurcations at $f_{1} \approx 0.06032$ and 0.06107 on the $\mathrm{TS}_{\pi}$ and $\mathrm{TS}_{0}$ branches; the profiles for the associated eigenfunctions of $\Xi_{\pi}$ and $\Xi_{0}$, respectively, show asymmetry perpendicular to the propagation direction as shown in Fig. 1(d). A plausible scenario is that rotational spot solutions for Eq. (14) described later originate in those pitchfork bifurcations. On the other hand, it is confirmed in the reduced ODEs that RS solutions emerge from such bifurcation points as is discussed below. The solution of Eq. (13) becomes unstable when the coefficient of the angle equation of Eq. (12) is positive. That is, the following solutions of Eq. (15) with $|\cos \theta| \neq 1$ emanate via pitchfork bifurcation,

$$
Q^{2}=\left(-\frac{2 \beta}{\alpha}\right) S^{2}=\left(-\frac{2 \beta}{\alpha}\right) \frac{N_{3}+2 M_{3}}{K},
$$

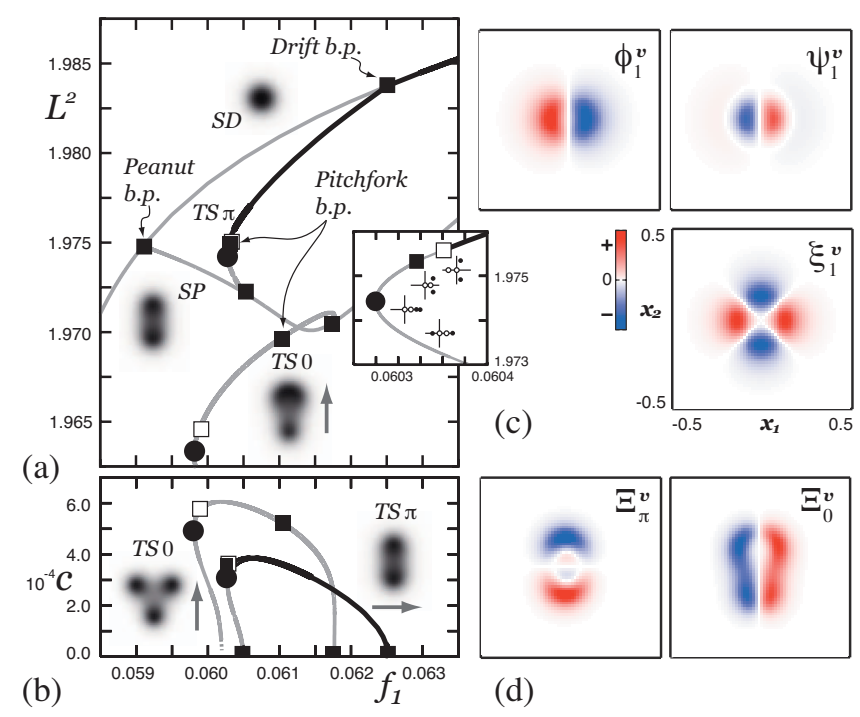

FIG. 1. (Color) (a) Bifurcation diagram of traveling spot solutions in the PDE system of Eq. (11). The solid and gray lines indicate the stable and unstable solutions. The corresponding traveling velocity $c$ is shown in (b). The black and white squares indicate the pitchfork and Hopf points and the black disks show the saddle-node points, respectively. The inset shows the magnified saddle-node point for $\mathrm{TS}_{\pi}$ branch with eigenvalues distribution. Just before this point, the Hopf and pitchfork bifurcations occur. Note that the tip splitting occurs for $\mathrm{TS}_{0}$ solutions via a saddle-node bifurcation, and it is expected that its branch ends up to the stationary $\mathcal{D}_{3}$ spot. (c) Profiles of eigenfunctions of standing disk spot solutions at the bifurcation points: translation-free mode $\phi_{1}$ and deformation vector $\psi_{1}$ at the drift bifurcation point; peanut mode $\xi_{1}$ at the peanut bifurcation point. (d) Profiles of the symmetry-breaking eigenfunctions of $\Xi_{\pi}$ and $\Xi_{0}$ at the pitchfork bifurcation points for $\mathrm{TS}_{\pi}$ and $\mathrm{TS}_{0}$ solutions. Only the $v$ component is shown here. Spectral computations are done with the system size $1.5 \times 1.5$.

$$
\cos ^{2} \theta=\frac{\left[N_{3}\left(M_{2}-2 \beta M_{1} / \alpha\right)-M_{3}\left(N_{1}-2 \beta N_{2} / \alpha\right)\right]^{2}}{\beta^{2}\left(N_{3}+2 M_{3}\right) K},
$$

where $K=4 \beta M_{1} / \alpha-2 M_{2}-N_{1}+2 \beta N_{2} / \alpha$. Accordingly, we solve the slave part in Eq. (9) as $z_{0}$ $=(2 / \alpha \beta)^{1 / 2}\left(\beta^{\prime} S e^{i \theta_{0}}-1\right) e^{i \beta S} \sin \theta t / \sin \theta$, where $\theta_{0}$ is constant. This allows the occurrence of RS motion with radius $\left|z_{0}\right|^{2}=2\left[\left(\beta^{\prime} S\right)^{2}-1\right] /\left(\alpha \beta \sin ^{2} \theta\right)$ for $\cos \theta_{0}=\left(\beta^{\prime} S\right)^{-1}$. Since the phase speed $\dot{\psi}=2 \dot{\phi}=2 \beta S \sin \theta$ becomes zero at the pitchfork bifurcation point of $|\cos \theta|=1$, where $Q$ and $\theta$ are continuous, clockwise and counterclockwise rotational motions with an infinite radius are equally possible to emanate from a straight motion. Here we consider $\alpha \beta<0$ which is numerically confirmed as $\alpha \approx-31.8, \beta \approx 1.0$, and $\beta^{\prime} \approx-326.7$ from Eq. (8). The constants of Eqs. (10) and (11) are also computed as $M_{1} \approx-61.3, \quad M_{2} \approx-3.9, \quad N_{1} \approx-240.0, \quad$ and $N_{2} \approx-35.6$, for which the eigenfunctions are normalized to satisfy the conditions of Eq. (2). Figures 2(a)-2(c) show the RS solution for the ODE dynamics of Eq. (9), in which the other parameter values are set to $\left(M_{3}, N_{3}\right)=(0.02,0.1)$. A bifurcation leading to the onset of RS motion is also shown in 

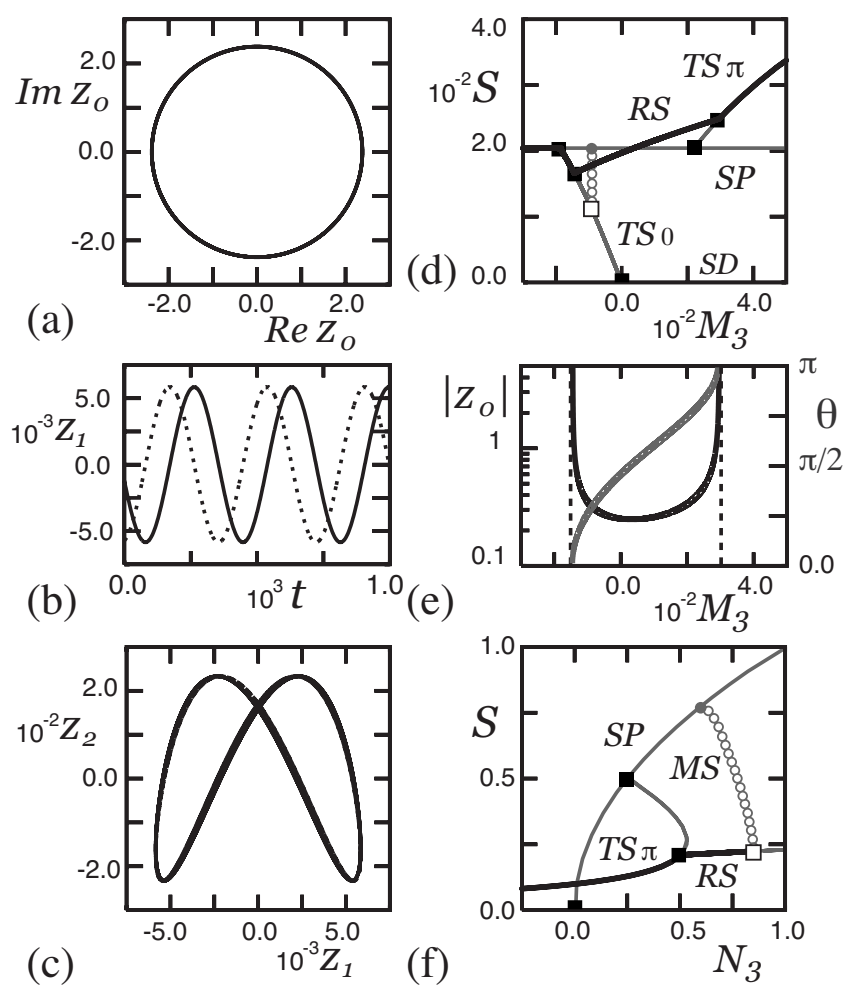

(f)

FIG. 2. (a,b,c) 1:2 mode interaction in a rotational spot (RS) motion for ODE of Eq. (6). Real (solid line) and imaginary (dotted line) parts are shown in (b) and (c). (d) Bifurcation diagram of spot solutions for the ODEs of Eq. (9), where $N_{3}$ is fixed to 0.1. Stable RS motion appears via pitchfork bifurcations and connects between the $\mathrm{TS}_{0}$ and $\mathrm{TS}_{\pi}$ branches. White disk indicates the unstable breather solution. (e) Radius $\left|z_{0}\right|$ (solid line) and angle $\theta$ (gray line) change of RS motion. (f) RS motion loses its stability via Torus bifurcation and MS motion (white disk) emanates, where $M_{3}$ is fixed to 1 .

Figs. 2(d) and 2(e). A stable RS branch emanates from pitchfork bifurcations on the TS branches and connects smoothly between the two types of straight motions of $\mathrm{TS}_{0}$ and $\mathrm{TS}_{\pi}$. The system of Eq. (12) inherits the variety of spot dynamics associated with global behaviors of bifurcation branches, including the hidden unstable branches. We assume that $M_{1}$, $M_{2}, N_{1}$, and $N_{2}$ are negative and either $M_{3}$ or $N_{3}$ is positive. Their values can be tuned up to realize the saddle-node structure of the $\mathrm{TS}_{\pi}$ branch, which is the case for the PDE structure of Fig. 1(a), by analyzing Eq. (13) for $\left(M_{1}, M_{2}, N_{1}, N_{2}\right)=(-8,-2,-1,-10)$ as shown in Fig. 2(f). More systematic calculations of bifurcational structures for 1:2 mode interaction dynamics were carried out by Holmes et al. $[16,17]$.

In searching the PDE dynamics for the parameter region close to the saddle-node point in Fig. 1(a), we find the RS motion for $f_{1} \in$ [0.06031:0.06036]. Its trajectory of centroid of $v$-component distribution draws a circle as shown in Fig. 3(a). Figures 3(b) and 3(c) show the spatiotemporal patterns of the $v$-component profiles along the trace of centroids and circular mapping plot $v(t, \phi)$. A spot almost maintains the shape and rotates with constant velocity. However, there is a small internal breathing motion as the time variation in $L^{2}$ slightly oscillates four times during a rotation as shown in
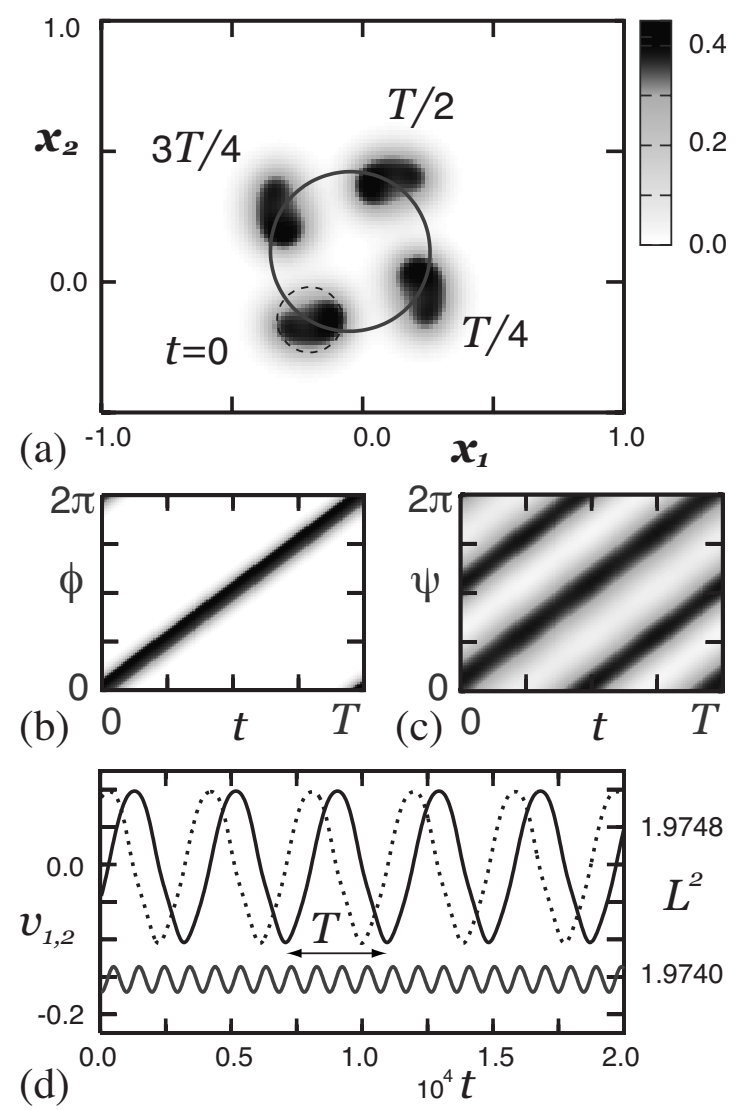

FIG. 3. Rotational spot (RS) motion in the PDE system of Eq. (11). (a) A spot moves in a counterclockwise direction as observed in four superimposed snapshots at $f_{1} \approx 0.06035$. The trajectory of the centroid of the $v$-component distribution is depicted by the solid line. The radius of RS motion is estimated as 0.31. (b) Spatiotemporal pattern of the $v$-component profile $v(t, \phi)$ along the trace of the centroid. (c) Circular mapping of $v(t, \psi)$ as the radial profile of $v$ component from the centroid, as indicated by the dotted circle in (a). $\psi=0$ is fixed in the $x$ axis and the radius is set to 0.125 . (d) Time evolutions of propagation velocity components $\left(v_{1}, v_{2}\right)$ and $L^{2}$ norms are shown by solid, dotted, and gray lines, respectively. We estimate the time periods for RS motion as $T \approx 3.9 \times 10^{3}$. Computations are carried out with system size $4 \times 4$ subject to the periodic boundary condition. The grid sizes are $\Delta x=\Delta y=2^{-6}$ and $\Delta t=0.10$.

Fig. 3(d). When $f_{1}$ decreases, both radius and period of RS motion decrease. As $f_{1}$ continues to decrease, modulatory instability occurs and grows, resulting in a spot splitting behavior. Investigation of the linearization of Eq. (12) shows that the RS solution of Eq. (15) loses its stability via Torus bifurcation and unstable modulatory spot motion, for which both amplitude and phase change in time, may emanate and end up with a heteroclinic bifurcation on the SP branch. The details are left for future work.

In summary, we have studied the localized spot dynamics near the drift and peanut codimension 2 singularity in a class of three-component reaction-diffusion systems. Interaction between the $\mathcal{D}_{1}$ and $\mathcal{D}_{2}$ symmetry-breaking deformations viewed from a $\mathcal{D}_{\infty}$ shape of a $\mathrm{SD}$ spot is analogous to $1: 2$ resonance patterns. It turns out that the corresponding bifurcations and the resulting straight motions of $\mathrm{TS}_{0}$ and $\mathrm{TS}_{\pi}$ are 
crucial for understanding the onset of rotational motion of traveling spots in two dimensions.

\section{ACKNOWLEDGMENTS}

We would like to thank Professor S.-I. Ei for valuable comments. This work was partially supported by the Grantin-Aid for Scientific Research under Grant No. (B)21340019 and the Japanese Ministry of Education, Science, Sports and Culture, Grant-in-Aid for Young Scientists under Grant No. (B) 20740224.

\section{APPENDIX A: CONSTANTS $M_{1}, M_{2}$, AND $M_{3}$}

In the appendix, we will give the derivations of Eqs. (10) and (11). The basic idea is shown in [13], however, it is technically complicated to derive their explicit form for codimension 2 singularity in two dimensions.

Since $(\tau(\boldsymbol{p}) \boldsymbol{u})_{t}=-\tau(\boldsymbol{p})\langle\dot{\boldsymbol{p}}, \nabla \boldsymbol{u}\rangle_{L^{2}}$ holds, we have

$$
\begin{aligned}
\boldsymbol{u}_{t} & =-\tau(\boldsymbol{p})\langle\dot{\boldsymbol{p}}, \nabla \boldsymbol{u}\rangle_{L^{2}}+\tau(\boldsymbol{p})\left\{\sum_{i=1}^{2} \dot{q}_{i} \boldsymbol{\psi}_{i}+\sum_{i=1}^{2} \dot{s}_{i} \boldsymbol{\xi}_{i}\right\} \\
& =\tau(\boldsymbol{p})\left\{-\langle\dot{\boldsymbol{p}}, \nabla \boldsymbol{u}\rangle_{L^{2}}+\sum_{i=1}^{2} \dot{q}_{i} \boldsymbol{\psi}_{i}+\sum_{i=1}^{2} \dot{s}_{i} \boldsymbol{\xi}_{i}\right\},
\end{aligned}
$$

where $\quad\langle\dot{\boldsymbol{p}}, \nabla \boldsymbol{u}\rangle_{L^{2}}=\langle\boldsymbol{p}, \nabla S\rangle_{L^{2}}+q_{1}\left\langle\dot{\boldsymbol{p}}, \nabla \boldsymbol{\psi}_{1}\right\rangle_{L^{2}}+q_{2}\left\langle\dot{\boldsymbol{p}}, \nabla \boldsymbol{\psi}_{2}\right\rangle_{L^{2}}$ $+s_{1}\left\langle\dot{\boldsymbol{p}}, \nabla \boldsymbol{\xi}_{1}\right\rangle_{L^{2}}+s_{2}\left\langle\dot{\boldsymbol{p}}, \nabla \boldsymbol{\xi}_{2}\right\rangle_{L^{2}}+\left\langle\dot{\boldsymbol{p}}, \nabla \boldsymbol{\zeta}^{\dagger}\right\rangle_{L^{2}}$ and

$$
\begin{aligned}
\mathcal{L}(\boldsymbol{u})+ & \sum_{i=1}^{2} \eta_{i} \boldsymbol{g}_{i}(\boldsymbol{u}) \\
= & \tau(P)\left\{-\sum_{i=1}^{2} q_{i} \boldsymbol{\phi}_{i}+L \boldsymbol{\zeta}^{\dagger}+\frac{1}{2} F^{\prime \prime}(S) \boldsymbol{W}^{2}+\frac{1}{6} F^{\prime \prime \prime}(S) \boldsymbol{W}^{3}\right. \\
& \left.+\sum_{i=1}^{2} \eta_{i} \boldsymbol{g}_{i}(S)+\sum_{i=1}^{2} \eta_{i} \boldsymbol{g}_{i}^{\prime}(S) \boldsymbol{W}\right\},
\end{aligned}
$$

where $\boldsymbol{W}=\sum_{i=1}^{2} q_{i} \boldsymbol{\psi}_{i}(\boldsymbol{r})+\sum_{i=1}^{2} s_{i} \boldsymbol{\xi}_{i}(\boldsymbol{r})+\boldsymbol{\zeta}^{\dagger}$. Here we will convert the equation of Eq. (1) of $\boldsymbol{u}$ to that of $(\boldsymbol{p}, \boldsymbol{q}, \boldsymbol{s})$. Taking the inner products with $\boldsymbol{\psi}_{i}^{*}(i=1,2)$, we have

$$
\begin{aligned}
& \left\langle\boldsymbol{u}_{t}, \boldsymbol{\psi}_{1}^{*}\right\rangle_{L^{2}}=-\dot{p}_{1}\left\langle\boldsymbol{\phi}_{1}, \boldsymbol{\psi}_{1}^{*}\right\rangle_{L^{2}}-\dot{p}_{1} s_{1}\left\langle\boldsymbol{\xi}_{1 x_{1}}, \boldsymbol{\psi}_{1}^{*}\right\rangle_{L^{2}} \\
& -\dot{p}_{2} s_{2}\left\langle\boldsymbol{\xi}_{2 x_{2}}, \boldsymbol{\psi}_{1}^{*}\right\rangle_{L^{2}} \\
& \left\langle\mathcal{L}(\boldsymbol{u})+\sum_{i=1}^{2} \eta_{i} \boldsymbol{g}_{i}(\boldsymbol{u}), \boldsymbol{\psi}_{1}^{*}\right\rangle_{L^{2}}=-q_{1}\left\langle\boldsymbol{\phi}_{1}, \boldsymbol{\psi}_{1}^{*}\right\rangle_{L^{2}} \\
& +q_{1} s_{1}\left\langle L \boldsymbol{\zeta}_{7}+F^{\prime \prime}(S) \boldsymbol{\psi}_{1} \boldsymbol{\xi}_{1}, \boldsymbol{\psi}_{1}^{*}\right\rangle_{L^{2}} \\
& +q_{2} s_{2}\left\langle L \boldsymbol{\zeta}_{8}+F^{\prime \prime}(S) \boldsymbol{\psi}_{2} \boldsymbol{\xi}_{2}, \boldsymbol{\psi}_{1}^{*}\right\rangle_{L^{2}}, \\
& \begin{aligned}
\left\langle\boldsymbol{u}_{t}, \boldsymbol{\psi}_{2}^{*}\right\rangle_{L^{2}}= & -\dot{p}_{2}\left\langle\boldsymbol{\phi}_{2}, \boldsymbol{\psi}_{2}^{*}\right\rangle_{L^{2}}-\dot{p}_{1} s_{2}\left\langle\boldsymbol{\xi}_{2 x_{1}}, \boldsymbol{\psi}_{2}^{*}\right\rangle_{L^{2}} \\
& -\dot{p}_{2} s_{1}\left\langle\boldsymbol{\xi}_{1 x_{2}}, \boldsymbol{\psi}_{2}^{*}\right\rangle_{L^{2}},
\end{aligned}
\end{aligned}
$$

$$
\begin{aligned}
\left\langle\mathcal{L}(\boldsymbol{u})+\sum_{i=1}^{2} \eta_{i} \boldsymbol{g}_{i}(\boldsymbol{u}), \boldsymbol{\psi}_{2}^{*}\right\rangle_{L^{2}} & -q_{2}\left\langle\boldsymbol{\phi}_{2}, \boldsymbol{\psi}_{2}^{*}\right\rangle_{L^{2}} \\
& +q_{1} s_{2}\left\langle L \boldsymbol{\zeta}_{9}+F^{\prime \prime}(S) \boldsymbol{\psi}_{1} \boldsymbol{\xi}_{2}, \boldsymbol{\psi}_{2}^{*}\right\rangle_{L^{2}} \\
& +q_{2} s_{1}\left\langle L \boldsymbol{\zeta}_{10}\right. \\
& \left.+F^{\prime \prime}(S) \boldsymbol{\psi}_{2} \boldsymbol{\xi}_{1}, \boldsymbol{\psi}_{2}^{*}\right\rangle_{L^{2}},
\end{aligned}
$$

where we show only nonzero terms. Since $\boldsymbol{\psi}_{1}=\cos \theta \psi(r)$, $\boldsymbol{\phi}_{1}=\cos \theta \phi(r)$, and $\boldsymbol{\xi}_{1}=\cos 2 \theta \xi(r)$ and so on, we can rewrite Eq. (8) as

$$
\begin{gathered}
\left\langle F^{\prime \prime}(S) \psi^{2}+2\left(\psi_{r}-\frac{\psi}{r}\right)-2 \alpha \xi, \xi^{*}\right\rangle=0, \\
\left\langle F^{\prime \prime}(S) \psi \xi+\xi_{r}+\frac{2 \xi}{r}-\beta \psi-\beta^{\prime} \phi, \phi^{*}\right\rangle=0, \\
\left\langle F^{\prime \prime}(S) \psi \xi+\xi_{r}+\frac{2 \xi}{r}-\beta \psi-\beta^{\prime} \phi, \psi^{*}\right\rangle=0 .
\end{gathered}
$$

We know that $\dot{p}_{1}=q_{1}-\beta^{\prime}\left(q_{1} s_{1}+q_{2} s_{2}\right)$ and $\dot{p}_{2}=q_{2}-\beta^{\prime}\left(q_{1} s_{2}\right.$ $\left.-q_{2} s_{1}\right)$, respectively. Next, in order to obtain the equation of motion of $q_{i}$, we take the inner products with $\boldsymbol{\phi}_{i}^{*}(i=1,2)$ as

$$
\begin{aligned}
\left\langle\boldsymbol{u}_{t}, \boldsymbol{\phi}_{1}^{*}\right\rangle_{L^{2}} & =-\dot{p}_{1}\left\langle\boldsymbol{\zeta}_{x_{1}}^{\dagger}, \boldsymbol{\phi}_{1}^{*}\right\rangle_{L^{2}}+\dot{q}_{1}\left\langle\boldsymbol{\psi}_{1}, \boldsymbol{\phi}_{1}^{*}\right\rangle_{L^{2}} \\
& =\left\langle\mathcal{L}(\boldsymbol{u})+\sum_{i=1}^{2} \eta_{i} \boldsymbol{g}_{i}(\boldsymbol{u}), \boldsymbol{\phi}_{1}^{*}\right\rangle_{L^{2}}, \\
\left\langle\boldsymbol{u}_{t}, \boldsymbol{\phi}_{2}^{*}\right\rangle_{L^{2}} & =-\dot{p}_{2}\left\langle\boldsymbol{\zeta}_{x_{2}}^{\dagger}, \boldsymbol{\phi}_{2}^{*}\right\rangle_{L^{2}}+\dot{q}_{2}\left\langle\boldsymbol{\psi}_{2}, \boldsymbol{\phi}_{2}^{*}\right\rangle_{L^{2}} \\
& =\left\langle\mathcal{L}(\boldsymbol{u})+\sum_{i=1}^{2} \eta_{i} \boldsymbol{g}_{i}(\boldsymbol{u}), \boldsymbol{\phi}_{2}^{*}\right\rangle_{L^{2}} .
\end{aligned}
$$

Computing each term of Eq. (A3), the first term of $M_{1}$ is given as

$$
\begin{aligned}
\frac{1}{6}\left\langle F^{\prime \prime \prime}(S) \boldsymbol{\psi}_{1}^{3}, \boldsymbol{\phi}_{1}^{*}\right\rangle_{L^{2}} & =\frac{1}{6} \int_{0}^{2 \pi} \cos ^{4} \theta d \theta \int_{0}^{\infty} r\left\langle F^{\prime \prime \prime}(S) \psi^{3}, \phi^{*}\right\rangle d r \\
& =\frac{\pi}{8} \int_{0}^{\infty} r\left\langle F^{\prime \prime \prime}(S) \psi^{3}, \phi^{*}\right\rangle d r
\end{aligned}
$$

By similar calculations to the above, we have

$$
\begin{aligned}
\frac{1}{6}\left\langle F^{\prime \prime \prime}(S) \boldsymbol{\psi}_{1}^{3}, \boldsymbol{\phi}_{1}^{*}\right\rangle_{L^{2}} & =\frac{1}{2}\left\langle F^{\prime \prime \prime}(S) \boldsymbol{\psi}_{1} \boldsymbol{\psi}_{2}^{2}, \boldsymbol{\phi}_{1}^{*}\right\rangle_{L^{2}} \\
& =\frac{1}{6}\left\langle F^{\prime \prime \prime}(S) \boldsymbol{\psi}_{2}^{3}, \boldsymbol{\phi}_{2}^{*}\right\rangle_{L^{2}} \\
& =\frac{1}{2}\left\langle F^{\prime \prime \prime}(S) \boldsymbol{\psi}_{1}^{2} \boldsymbol{\psi}_{2}, \boldsymbol{\phi}_{2}^{*}\right\rangle_{L^{2}} \equiv \pi M_{1}^{\prime}
\end{aligned}
$$

We can rewrite Eq. (4) as

$$
L \zeta_{1}+\frac{1}{2} F^{\prime \prime}(S) \cos ^{2} \theta \psi^{2}+\cos ^{2} \theta \psi_{r}+\sin ^{2} \theta \frac{\psi}{r}=\alpha \cos 2 \theta \xi
$$




$$
\begin{gathered}
L \zeta_{2}+\frac{1}{2} F^{\prime \prime}(S) \sin ^{2} \theta \psi^{2}+\sin ^{2} \theta \psi_{r}+\cos ^{2} \theta \frac{\psi}{r}=-\alpha \cos 2 \theta \xi \\
L \zeta_{3}+F^{\prime \prime}(S) \sin \theta \cos \theta \psi^{2}+2 \sin \theta \cos \theta\left(\psi_{r}-\frac{\psi}{r}\right) \\
=2 \alpha \sin 2 \theta \xi
\end{gathered}
$$

The term of $\boldsymbol{\zeta}_{1}-\boldsymbol{\zeta}_{2}$ satisfies

$$
\begin{aligned}
-L\left(\boldsymbol{\zeta}_{1}-\zeta_{2}\right)= & \frac{1}{2}\left(\cos ^{2} \theta-\sin ^{2} \theta\right)\left[F^{\prime \prime}(S) \psi^{2}+2\left(\psi_{r}-\frac{\psi}{r}\right)\right] \\
& -2 \alpha \cos 2 \theta \xi \\
= & \frac{1}{2} \sin \left(2 \theta+\frac{\pi}{2}\right)\left[F^{\prime \prime}(S) \psi^{2}+2\left(\psi_{r}-\frac{\psi}{r}\right)-4 \alpha \xi\right] .
\end{aligned}
$$

Hence we have $\left(\boldsymbol{\zeta}_{1}-\zeta_{2}\right)(r, \theta)=\boldsymbol{\zeta}_{3}\left(r, \theta+\frac{\pi}{4}\right)$. We note that $\boldsymbol{\zeta}_{1}$ $=\cos ^{2} \theta \widetilde{\zeta}_{1}(r)+\sin ^{2} \theta \widetilde{\zeta}_{2}(r), \quad \zeta_{2}=\sin ^{2} \theta \widetilde{\zeta}_{1}(r)+\cos ^{2} \theta \widetilde{\zeta}_{2}(r)$, and $\zeta_{3}=\sin 2 \theta \widetilde{\zeta}_{3}(r)$ hold. It is easy to see that $\zeta_{2}(r, \theta)$ $=\zeta_{1}\left(r, \theta+\frac{\pi}{2}\right)$. Thus, the second term of $M_{1}$ has the following:

$$
\begin{aligned}
\left\langle F^{\prime \prime}(S) \psi_{1} \zeta_{1}, \boldsymbol{\phi}_{1}^{*}\right\rangle_{L^{2}}= & \int_{0}^{2 \pi} \int_{0}^{\infty} \cos ^{2} \theta r\left\langle F^{\prime \prime}(S) \psi \zeta_{1}, \phi^{*}\right\rangle d r d \theta \\
= & \frac{3 \pi}{4} \int_{0}^{\infty} r\left\langle F^{\prime \prime} \psi \widetilde{\zeta}_{1}, \phi^{*}\right\rangle d r \\
& +\frac{\pi}{4} \int_{0}^{\infty} r\left\langle F^{\prime \prime} \psi \widetilde{\zeta}_{2}, \phi^{*}\right\rangle d r
\end{aligned}
$$

Similarly, we have

$$
\begin{aligned}
\left\langle F^{\prime \prime}(S) \boldsymbol{\psi}_{1} \zeta_{1}, \boldsymbol{\phi}_{1}^{*}\right\rangle_{L^{2}} & =\left\langle F^{\prime \prime}(S) \boldsymbol{\psi}_{1} \boldsymbol{\zeta}_{2}, \boldsymbol{\phi}_{1}^{*}\right\rangle_{L^{2}}+\left\langle F^{\prime \prime}(S) \boldsymbol{\psi}_{2} \boldsymbol{\zeta}_{3}, \boldsymbol{\phi}_{1}^{*}\right\rangle_{L^{2}} \\
& =\left\langle F^{\prime \prime}(S) \boldsymbol{\psi}_{2} \boldsymbol{\zeta}_{2}, \boldsymbol{\phi}_{2}^{*}\right\rangle_{L^{2}} \\
& =\left\langle F^{\prime \prime}(S) \boldsymbol{\psi}_{2} \boldsymbol{\zeta}_{1}, \boldsymbol{\phi}_{2}^{*}\right\rangle_{L^{2}}+\left\langle F^{\prime \prime}(S) \boldsymbol{\psi}_{1} \zeta_{3}, \boldsymbol{\phi}_{2}^{*}\right\rangle_{L^{2}} \\
& \equiv \pi M_{1}^{\prime \prime},
\end{aligned}
$$

where we use the relation of $\tilde{\zeta}_{1}(r)-\widetilde{\zeta}_{2}(r)=\widetilde{\zeta}_{3}(r)$. The last term of $M_{1}$ is obtained as

$$
\begin{aligned}
\left\langle\boldsymbol{\zeta}_{1 x_{1}}, \boldsymbol{\phi}_{1}^{*}\right\rangle_{L^{2}}= & \int_{0}^{2 \pi} \int_{0}^{\infty} \cos ^{2} \theta r\left\langle\boldsymbol{\zeta}_{1 r}, \phi^{*}\right\rangle d r d \theta \\
& -\int_{0}^{2 \pi} \int_{0}^{\infty} \sin \theta \cos \theta\left\langle\zeta_{1 \theta}, \boldsymbol{\phi}^{*}\right\rangle d r d \theta \\
= & \frac{3 \pi}{4} \int_{0}^{\infty} r\left\langle\widetilde{\zeta}_{1 r}, \phi^{*}\right\rangle d r+\frac{\pi}{4} \int_{0}^{\infty} r\left\langle\widetilde{\zeta}_{2 r}, \phi^{*}\right\rangle d r \\
& -\frac{\pi}{2} \int_{0}^{\infty}\left\langle\widetilde{\zeta}_{2}-\widetilde{\zeta}_{1}, \phi^{*}\right\rangle d r .
\end{aligned}
$$

Here, we also have

$$
\begin{aligned}
\left\langle\boldsymbol{\zeta}_{1 x_{1}}, \boldsymbol{\phi}_{1}^{*}\right\rangle_{L^{2}}-\left\langle\boldsymbol{\zeta}_{2 x_{1}}, \boldsymbol{\phi}_{1}^{*}\right\rangle_{L^{2}}-\left\langle\boldsymbol{\zeta}_{3 x_{2}}, \boldsymbol{\phi}_{1}^{*}\right\rangle_{L^{2}} & \\
= & \frac{\pi}{2} \int_{0}^{\infty} r\left\langle\widetilde{\zeta}_{1 r}, \boldsymbol{\phi}^{*}\right\rangle d r-\frac{\pi}{2} \int_{0}^{\infty} r\left\langle\widetilde{\zeta}_{2 r}, \phi^{*}\right\rangle d r \\
& +\pi \int_{0}^{\infty}\left\langle\widetilde{\zeta}_{1}-\widetilde{\zeta}_{2}, \phi^{*}\right\rangle d r-\frac{\pi}{2} \int_{0}^{\infty} r\left\langle\widetilde{\zeta}_{3 r}, \phi^{*}\right\rangle d r \\
& -\pi \int_{0}^{\infty}\left\langle\widetilde{\zeta}_{3}, \phi^{*}\right\rangle d r=0 .
\end{aligned}
$$

In view of the above results, we have

$$
\begin{aligned}
\left\langle\boldsymbol{\zeta}_{1 x_{1}}, \boldsymbol{\phi}_{1}^{*}\right\rangle_{L^{2}} & =\left\langle\boldsymbol{\zeta}_{2 x_{1}}, \boldsymbol{\phi}_{1}^{*}\right\rangle_{L^{2}}+\left\langle\boldsymbol{\zeta}_{3 x_{2}}, \boldsymbol{\phi}_{1}^{*}\right\rangle_{L^{2}} \\
& =\left\langle\boldsymbol{\zeta}_{2 x_{2}}, \boldsymbol{\phi}_{2}^{*}\right\rangle_{L^{2}} \\
& =\left\langle\boldsymbol{\zeta}_{1 x_{2}}, \boldsymbol{\phi}_{2}^{*}\right\rangle_{L^{2}}+\left\langle\boldsymbol{\zeta}_{3 x_{1}}, \boldsymbol{\phi}_{2}^{*}\right\rangle_{L^{2}} \\
& \equiv \pi M_{1}^{\prime \prime \prime}
\end{aligned}
$$

In this way, we obtain $M_{1}=M_{1}^{\prime}+M_{1}^{\prime \prime}+M_{1}^{\prime \prime \prime}$.

Let us now rewrite Eq. (5) as

$$
\begin{gathered}
L \zeta_{4}+\frac{1}{2} F^{\prime \prime}(S) \cos ^{2} 2 \theta \xi^{2}=0, \\
L \zeta_{5}+\frac{1}{2} F^{\prime \prime}(S) \sin ^{2} 2 \theta \xi^{2}=0, \\
L \zeta_{6}+F^{\prime \prime}(S) \sin 2 \theta \cos 2 \theta \xi^{2}=0
\end{gathered}
$$

We note that $\zeta_{4}=\cos ^{2} 2 \theta \widetilde{\zeta}_{4}, \zeta_{5}=\sin ^{2} 2 \theta \tilde{\zeta}_{5}$, and $\zeta_{6}=\sin 4 \theta \tilde{\zeta}_{6}$. It is easy to see that $\zeta_{5}(r, \theta)=\zeta_{4}\left(r, \theta+\frac{\pi}{4}\right)$. The term of $\boldsymbol{\zeta}_{4}-\boldsymbol{\zeta}_{5}$ satisfies

$$
\begin{aligned}
-L\left(\zeta_{4}-\zeta_{5}\right) & =\frac{1}{2}\left(\cos ^{2} 2 \theta-\sin ^{2} 2 \theta\right) F^{\prime \prime}(S) \xi^{2} \\
& =\frac{1}{2} \sin \left(4 \theta+\frac{\pi}{2}\right) F^{\prime \prime}(S) \xi^{2} .
\end{aligned}
$$

Hence we have $\left(\boldsymbol{\zeta}_{4}-\zeta_{5}\right)(r, \theta)=\zeta_{6}\left(r, \theta+\frac{\pi}{8}\right)$. We can rewrite Eq. (6) as

$$
\begin{aligned}
L \zeta_{7} & +F^{\prime \prime}(S) \cos \theta \cos 2 \theta \psi \xi+\cos \theta \cos 2 \theta \xi_{r}+\sin \theta \sin 2 \theta \frac{2 \xi}{r} \\
& =\cos \theta\left(\beta \psi+\beta^{\prime} \phi\right),
\end{aligned}
$$

$L \zeta_{8}+F^{\prime \prime}(S) \sin \theta \sin 2 \theta \psi \xi+\sin \theta \sin 2 \theta \xi_{r}+\cos \theta \cos 2 \theta \frac{2 \xi}{r}$ $=\cos \theta\left(\beta \psi+\beta^{\prime} \phi\right)$,

$L \zeta_{9}+F^{\prime \prime}(S) \cos \theta \sin 2 \theta \psi \xi+\cos \theta \sin 2 \theta \xi_{r}-\sin \theta \cos 2 \theta \frac{2 \xi}{r}$ $=\sin \theta\left(\beta \psi+\beta^{\prime} \phi\right)$, 


$$
\begin{aligned}
L \boldsymbol{\zeta}_{10} & +F^{\prime \prime}(S) \sin \theta \cos 2 \theta \psi \xi+\sin \theta \cos 2 \theta \xi_{r} \\
& -\cos \theta \sin 2 \theta \frac{2 \xi}{r}=-\sin \theta\left(\beta \psi+\beta^{\prime} \phi\right) .
\end{aligned}
$$

We note that $\zeta_{7}=\cos \theta \cos 2 \theta \widetilde{\zeta}_{7}+\sin \theta \sin 2 \theta \widetilde{\zeta}_{8}$, $\zeta_{8}=\sin \theta \sin 2 \theta \widetilde{\zeta}_{7}+\cos \theta \cos 2 \theta \widetilde{\zeta}_{8}, \quad \zeta_{9}=\cos \theta \sin 2 \theta \widetilde{\zeta}_{9}$ $+\sin \theta \cos 2 \theta \widetilde{\zeta}_{10}$, and $\zeta_{10}=\sin \theta \cos 2 \theta \widetilde{\zeta}_{9}+\cos \theta \sin 2 \theta \widetilde{\zeta}_{10}$. The terms of $\zeta_{7} \pm \zeta_{8}$ satisfy

$$
-L\left(\boldsymbol{\zeta}_{7}+\boldsymbol{\zeta}_{8}\right)=(\cos \theta \cos 2 \theta+\sin \theta \sin 2 \theta)
$$

$$
\begin{aligned}
& \times\left(F^{\prime \prime}(S) \psi \xi+\xi_{r}-\frac{2 \xi}{r}\right)-2 \cos \theta\left(\beta \psi+\beta^{\prime} \phi\right) \\
= & \cos \theta\left(F^{\prime \prime}(S) \psi \xi+\xi_{r}-\frac{2 \xi}{r}-2\left(\beta \psi+\beta^{\prime} \phi\right)\right),
\end{aligned}
$$

$$
\begin{aligned}
-L\left(\boldsymbol{\zeta}_{7}-\zeta_{8}\right)= & (\cos \theta \cos 2 \theta-\sin \theta \sin 2 \theta) \\
& \times\left(F^{\prime \prime}(S) \psi \xi+\xi_{r}-\frac{2 \xi}{r}\right) \\
= & \cos 3 \theta\left(F^{\prime \prime}(S) \psi \xi+\xi_{r}-\frac{2 \xi}{r}\right),
\end{aligned}
$$

and the terms of $\zeta_{9} \pm \zeta_{10}$ satisfy

$$
\begin{aligned}
-L\left(\zeta_{9}+\zeta_{10}\right)= & (\cos \theta \sin 2 \theta+\sin \theta \cos 2 \theta) \\
& \times\left(F^{\prime \prime}(S) \psi \xi+\xi_{r}-\frac{2 \xi}{r}\right) \\
= & \sin 3 \theta\left(F^{\prime \prime}(S) \psi \xi+\xi_{r}-\frac{2 \xi}{r}\right), \quad(\mathrm{A} 19) \\
-L\left(\zeta_{9}-\zeta_{10}\right)= & (\cos \theta \sin 2 \theta-\sin \theta \cos 2 \theta) \\
& \times\left(F^{\prime \prime}(S) \psi \xi+\xi_{r}-\frac{2 \xi}{r}\right)-2 \sin \theta\left(\beta \psi+\beta^{\prime} \phi\right) \\
= & \sin \theta\left(F^{\prime \prime}(S) \psi \xi+\xi_{r}-\frac{2 \xi}{r}-2\left(\beta \psi+\beta^{\prime} \phi\right)\right) .
\end{aligned}
$$

Hence we have $\left(\boldsymbol{\zeta}_{7}+\boldsymbol{\zeta}_{8}\right)(r, \theta)=\left(\boldsymbol{\zeta}_{9}-\boldsymbol{\zeta}_{10}\right)\left(r, \theta+\frac{\pi}{2}\right)$ and $\left(\zeta_{7}-\zeta_{8}\right)(r, \theta)=\left(\zeta_{9}+\zeta_{10}\right)\left(r, \theta+\frac{\pi}{6}\right)$. The first three terms of $M_{2}$ are given as

$$
\begin{aligned}
& \left\langle\frac{1}{2} F^{\prime \prime \prime}(S) \boldsymbol{\xi}_{1}^{2} \boldsymbol{\psi}_{1}+F^{\prime \prime}(S) \boldsymbol{\psi}_{1} \boldsymbol{\zeta}_{4}+F^{\prime \prime}(S) \boldsymbol{\xi}_{1} \zeta_{7}, \boldsymbol{\phi}_{1}^{*}\right\rangle_{L^{2}} \\
& =\frac{1}{2} \int_{0}^{2 \pi} \cos ^{2} \theta \cos ^{2} 2 \theta d \theta \int_{0}^{\infty} r\left\langle F^{\prime \prime \prime}(S) \xi^{2} \psi, \phi^{*}\right\rangle d r \\
& \quad+\int_{0}^{2 \pi} \cos ^{2} \theta \cos ^{2} 2 \theta d \theta \int_{0}^{\infty} r\left\langle F^{\prime \prime}(S) \widetilde{\zeta}_{4} \psi, \phi^{*}\right\rangle d r \\
& \quad+\int_{0}^{2 \pi} \cos ^{2} \theta \cos ^{2} 2 \theta d \theta \int_{0}^{\infty} r\left\langle F^{\prime \prime}(S) \widetilde{\zeta}_{7} \xi, \phi^{*}\right\rangle d r
\end{aligned}
$$

$$
\begin{aligned}
& +\int_{0}^{2 \pi} \sin \theta \cos \theta \sin 2 \theta \cos 2 \theta d \theta \int_{0}^{\infty} r\left\langle F^{\prime \prime}(S) \tilde{\zeta}_{8} \xi, \phi^{*}\right\rangle d r \\
= & \frac{\pi}{4} \int_{0}^{\infty} r\left\langle F^{\prime \prime \prime}(S) \xi^{2} \psi, \phi^{*}\right\rangle d r+\frac{\pi}{2} \int_{0}^{\infty} r\left\langle F^{\prime \prime}(S) \tilde{\zeta}_{4} \psi, \phi^{*}\right\rangle d r \\
& +\frac{\pi}{2} \int_{0}^{\infty} r\left\langle F^{\prime \prime}(S) \tilde{\zeta}_{7} \xi, \phi^{*}\right\rangle d r .
\end{aligned}
$$

Quite similarly, we have

$$
\begin{aligned}
& \left\langle\frac{1}{2} F^{\prime \prime \prime}(S) \boldsymbol{\xi}_{1}^{2} \boldsymbol{\psi}_{1}+F^{\prime \prime}(S) \boldsymbol{\psi}_{1} \boldsymbol{\zeta}_{4}+F^{\prime \prime}(S) \boldsymbol{\xi}_{1} \boldsymbol{\zeta}_{7}, \boldsymbol{\phi}_{1}^{*}\right\rangle_{L^{2}} \\
& \quad\left\langle\frac{1}{2} F^{\prime \prime \prime}(S) \boldsymbol{\xi}_{1}^{2} \boldsymbol{\psi}_{2}+F^{\prime \prime}(S) \boldsymbol{\psi}_{2} \boldsymbol{\zeta}_{4}+F^{\prime \prime}(S) \boldsymbol{\xi}_{1} \zeta_{10}, \boldsymbol{\phi}_{2}^{*}\right\rangle_{L^{2}} \\
& \quad=\left\langle\frac{1}{2} F^{\prime \prime \prime}(S) \boldsymbol{\xi}_{2}^{2} \boldsymbol{\psi}_{2}+F^{\prime \prime}(S) \boldsymbol{\psi}_{2} \zeta_{5}+F^{\prime \prime}(S) \boldsymbol{\xi}_{2} \zeta_{8}, \boldsymbol{\phi}_{2}^{*}\right\rangle_{L^{2}} \\
& \quad\left\langle\frac{1}{2} F^{\prime \prime \prime}(S) \boldsymbol{\xi}_{2}^{2} \boldsymbol{\psi}_{1}+F^{\prime \prime}(S) \boldsymbol{\psi}_{1} \zeta_{5}+F^{\prime \prime}(S) \boldsymbol{\xi}_{2} \zeta_{9}, \boldsymbol{\phi}_{1}^{*}\right\rangle_{L^{2}} \equiv \pi M_{2}^{\prime} .
\end{aligned}
$$

Here we use $\widetilde{\zeta}_{4}(r)=\widetilde{\zeta}_{5}(r)=\widetilde{\zeta}_{6}(r)$ and $\widetilde{\zeta}_{7}(r)=\widetilde{\zeta}_{9}(r)$ and $\widetilde{\zeta}_{8}(r)=$ $-\tilde{\zeta}_{10}(r)$. The last two terms of $M_{2}$ are obtained as

$$
\begin{aligned}
\left\langle\boldsymbol{\zeta}_{4 x_{1}}, \boldsymbol{\phi}_{1}^{*}\right\rangle_{L^{2}}= & \int_{0}^{2 \pi} \cos ^{2} \theta \cos ^{2} 2 \theta d \theta \int_{0}^{\infty} r\left\langle\widetilde{\zeta}_{4 r}, \boldsymbol{\phi}^{*}\right\rangle d r \\
& +4 \int_{0}^{2 \pi} \sin \theta \cos \theta \sin 2 \theta \cos 2 \theta d \theta \\
& \times \int_{0}^{\infty}\left\langle\widetilde{\zeta}_{4}, \phi^{*}\right\rangle d r \\
= & \frac{\pi}{2} \int_{0}^{\infty} r\left\langle\widetilde{\zeta}_{4 r}, \phi^{*}\right\rangle d r
\end{aligned}
$$

and

$$
\begin{aligned}
\left\langle\boldsymbol{\xi}_{1 x_{1}}, \boldsymbol{\phi}_{1}^{*}\right\rangle_{L^{2}}= & \int_{0}^{2 \pi} \cos ^{2} \theta \cos 2 \theta d \theta \int_{0}^{\infty} r\left\langle\xi_{r}, \phi^{*}\right\rangle d r \\
& +2 \int_{0}^{2 \pi} \sin \theta \cos \theta \sin 2 \theta d \theta \int_{0}^{\infty}\left\langle\xi, \phi^{*}\right\rangle d r \\
= & \frac{\pi}{2} \int_{0}^{\infty} r\left\langle\xi_{r}, \phi^{*}\right\rangle d r+\pi \int_{0}^{\infty}\left\langle\xi, \phi^{*}\right\rangle d r .
\end{aligned}
$$

By similar calculations to the above, we have

$$
\begin{aligned}
& \left\langle\boldsymbol{\zeta}_{4 x_{1}}, \boldsymbol{\phi}_{1}^{*}\right\rangle_{L^{2}}=\left\langle\boldsymbol{\zeta}_{5 x_{2}}, \boldsymbol{\phi}_{2}^{*}\right\rangle_{L^{2}}=\left\langle\boldsymbol{\zeta}_{4 x_{2}}, \boldsymbol{\phi}_{2}^{*}\right\rangle_{L^{2}}=\left\langle\boldsymbol{\zeta}_{5 x_{1}}, \boldsymbol{\phi}_{1}^{*}\right\rangle_{L^{2}} \equiv \pi M_{2}^{\prime \prime} \\
& \text { and }
\end{aligned}
$$




$$
\begin{aligned}
\left\langle\boldsymbol{\xi}_{1 x_{1}}, \boldsymbol{\phi}_{1}^{*}\right\rangle_{L^{2}} & =\left\langle\boldsymbol{\xi}_{2 x_{1}}, \boldsymbol{\phi}_{2}^{*}\right\rangle_{L^{2}} \\
& =\left\langle\boldsymbol{\xi}_{2 x_{2}}, \boldsymbol{\phi}_{1}^{*}\right\rangle_{L^{2}} \\
& =-\left\langle\boldsymbol{\xi}_{1 x_{2}}, \boldsymbol{\phi}_{2}^{*}\right\rangle_{L^{2}} \\
& \equiv \pi M_{2}^{\prime \prime \prime}
\end{aligned}
$$

In this way, we obtain $M_{2}=M_{2}^{\prime}+M_{2}^{\prime \prime}-\beta^{\prime} M_{2}^{\prime \prime \prime}$. The terms of $M_{3}$ are shown quite similarly as

$$
\begin{aligned}
\left\langle F^{\prime \prime}(S) \boldsymbol{\psi}_{1} \zeta_{11}, \boldsymbol{\phi}_{1}^{*}\right\rangle_{L^{2}}+\left\langle\boldsymbol{g}_{1}^{\prime}(S) \boldsymbol{\psi}_{1}, \boldsymbol{\phi}_{1}^{*}\right\rangle_{L^{2}} \\
=\int_{0}^{2 \pi} \cos ^{2} \theta d \theta \int_{0}^{\infty} r\left\langle F^{\prime \prime}(S) \psi \zeta_{11}, \boldsymbol{\phi}^{*}\right\rangle d r \\
\quad+\int_{0}^{2 \pi} \cos ^{2} \theta d \theta \int_{0}^{\infty} r\left\langle\boldsymbol{g}_{1}^{\prime}(S) \psi, \boldsymbol{\phi}^{*}\right\rangle d r \\
=\pi \int_{0}^{\infty} r\left\langle F^{\prime \prime}(S) \psi \widetilde{\zeta}_{11}, \boldsymbol{\phi}^{*}\right\rangle d r+\pi \int_{0}^{\infty} r\left\langle\boldsymbol{g}_{1}^{\prime}(S) \psi, \boldsymbol{\phi}^{*}\right\rangle d r \\
=\left\langle F^{\prime \prime}(S) \boldsymbol{\psi}_{2} \boldsymbol{\zeta}_{11}, \boldsymbol{\phi}_{2}^{*}\right\rangle_{L^{2}}+\left\langle\boldsymbol{g}_{1}^{\prime}(S) \boldsymbol{\psi}_{2}, \boldsymbol{\phi}_{2}^{*}\right\rangle_{L^{2}} \equiv \pi M_{3}^{\prime} \quad(\mathrm{A} 27)
\end{aligned}
$$

and

$$
\begin{aligned}
\left\langle F^{\prime \prime}(S)\right. & \left.\boldsymbol{\psi}_{1} \zeta_{12}, \boldsymbol{\phi}_{1}^{*}\right\rangle_{L^{2}}+\left\langle\boldsymbol{g}_{2}^{\prime}(S) \boldsymbol{\psi}_{1}, \boldsymbol{\phi}_{1}^{*}\right\rangle_{L^{2}} \\
= & \int_{0}^{2 \pi} \cos ^{2} \theta d \theta \int_{0}^{\infty} r\left\langle F^{\prime \prime}(S) \psi \zeta_{12}, \boldsymbol{\phi}^{*}\right\rangle d r \\
& +\int_{0}^{2 \pi} \cos ^{2} \theta d \theta \int_{0}^{\infty} r\left\langle\boldsymbol{g}_{2}^{\prime}(S) \psi, \boldsymbol{\phi}^{*}\right\rangle d r \\
= & \pi \int_{0}^{\infty} r\left\langle F^{\prime \prime}(S) \psi \widetilde{\zeta}_{12}, \boldsymbol{\phi}^{*}\right\rangle d r+\pi \int_{0}^{\infty} r\left\langle\mathbf{g}_{2}^{\prime}(S) \psi, \boldsymbol{\phi}^{*}\right\rangle d r \\
= & \left\langle F^{\prime \prime}(S) \boldsymbol{\psi}_{2} \boldsymbol{\zeta}_{12}, \boldsymbol{\phi}_{2}^{*}\right\rangle_{L^{2}}+\left\langle\boldsymbol{g}_{2}^{\prime}(S) \boldsymbol{\psi}_{2}, \boldsymbol{\phi}_{2}^{*}\right\rangle_{L^{2}} \equiv \pi M_{3}^{\prime \prime} .
\end{aligned}
$$

Here $\zeta_{11}$ and $\zeta_{12}$ are radially symmetric as $\zeta_{11}=\widetilde{\zeta}_{11}(r)$ and $\zeta_{12}=\widetilde{\zeta}_{12}(r)$, respectively. The last term to $M_{3}$ is obtained as

$$
\begin{aligned}
\left\langle\boldsymbol{\zeta}_{11 x_{1}}, \boldsymbol{\phi}_{1}^{*}\right\rangle_{L^{2}} & =\int_{0}^{2 \pi} \cos ^{2} \theta d \theta \int_{0}^{\infty} r\left\langle\boldsymbol{\zeta}_{11 r}, \boldsymbol{\phi}^{*}\right\rangle d r \\
& =\pi \int_{0}^{\infty} r\left\langle\widetilde{\zeta}_{11 r}, \boldsymbol{\phi}^{*}\right\rangle d r \\
& =\left\langle\boldsymbol{\zeta}_{11 x_{2}}, \boldsymbol{\phi}_{2}^{*}\right\rangle_{L^{2}} \\
& \equiv \pi M_{3}^{\prime \prime \prime}
\end{aligned}
$$

$$
\begin{aligned}
\left\langle\boldsymbol{\zeta}_{12 x_{1}}, \boldsymbol{\phi}_{1}^{*}\right\rangle_{L^{2}} & =\int_{0}^{2 \pi} \cos ^{2} \theta d \theta \int_{0}^{\infty} r\left\langle\boldsymbol{\zeta}_{12 r}, \boldsymbol{\phi}^{*}\right\rangle d r \\
& =\pi \int_{0}^{\infty} r\left\langle\widetilde{\zeta}_{12 r}, \boldsymbol{\phi}^{*}\right\rangle d r \\
& =\left\langle\boldsymbol{\zeta}_{12 x_{2}}, \boldsymbol{\phi}_{2}^{*}\right\rangle_{L^{2}} \\
& \equiv \pi M_{3}^{\prime \prime \prime \prime}
\end{aligned}
$$

We arrive at the results of $M_{3}=\eta_{1}\left(M_{3}^{\prime}+M_{3}^{\prime \prime \prime}\right)+\eta_{2}\left(M_{3}^{\prime \prime}+M_{3}^{\prime \prime \prime \prime}\right)$. It is remarked that, for numerical computations of the constants, we shall normalize eigenfunctions of $\boldsymbol{\psi}_{i}^{0}, \boldsymbol{\psi}_{i}^{* 0}, \boldsymbol{\phi}_{i}^{* 0}$ obtained from numerical spectral analysis. According to Eq. (2), the eigenfunctions are given by

$$
\begin{gathered}
\boldsymbol{\psi}_{i}=\boldsymbol{\psi}_{i}^{0}-\frac{\left\langle\boldsymbol{\phi}_{i}, \boldsymbol{\psi}_{i}^{0}\right\rangle_{L^{2}}}{\left\langle\boldsymbol{\phi}_{i}, \boldsymbol{\phi}_{i}\right\rangle_{L^{2}}} \boldsymbol{\phi}_{i}, \\
\boldsymbol{\phi}_{i}^{*}=\frac{\pi}{\left\langle\boldsymbol{\phi}_{i}, \boldsymbol{\psi}_{i}^{* 0}\right\rangle_{L^{2}}} \boldsymbol{\phi}_{i}^{* 0},
\end{gathered}
$$

$$
\begin{aligned}
\boldsymbol{\psi}_{i}^{*}= & \frac{\pi}{\left\langle\boldsymbol{\phi}_{i}, \boldsymbol{\psi}_{i}^{* 0}\right\rangle_{L^{2}}} \boldsymbol{\psi}_{i}^{* 0}+\frac{\pi}{\left\langle\boldsymbol{\psi}_{i}^{0}, \boldsymbol{\phi}_{i}^{* 0}\right\rangle_{L^{2}}}\left(\frac{\left\langle\boldsymbol{\phi}_{i}, \boldsymbol{\psi}_{i}^{0}\right\rangle_{L^{2}}}{\left\langle\boldsymbol{\phi}_{i}, \boldsymbol{\phi}_{i}\right\rangle_{L^{2}}}\right. \\
& \left.-\frac{\left\langle\boldsymbol{\psi}_{i}^{0}, \boldsymbol{\psi}_{i}^{* 0}\right\rangle_{L^{2}}}{\left\langle\boldsymbol{\phi}_{i}, \boldsymbol{\psi}_{i}^{* 0}\right\rangle_{L^{2}}}\right) \boldsymbol{\phi}_{i}^{* 0} .
\end{aligned}
$$

\section{APPENDIX B: CONSTANTS $N_{1}, N_{2}$, AND $N_{3}$}

By similar calculations to those shown in the previous subsection, we have

$$
\begin{aligned}
&\left\langle F^{\prime \prime}(S) \boldsymbol{\xi}_{1} \boldsymbol{\zeta}_{11}, \boldsymbol{\xi}_{1}^{*}\right\rangle_{L^{2}}+\left\langle\boldsymbol{g}_{1}^{\prime}(S) \boldsymbol{\xi}_{1}, \boldsymbol{\xi}_{1}^{*}\right\rangle_{L^{2}} \int_{0}^{2 \pi} \cos ^{2} 2 \theta d \theta \int_{0}^{\infty} r\left\langle F^{\prime \prime}(S) \xi \xi_{11}, \xi^{*}\right\rangle d r \\
& \quad+\int_{0}^{2 \pi} \cos ^{2} 2 \theta d \theta \int_{0}^{\infty} r\left\langle\boldsymbol{g}_{1}^{\prime}(S) \xi, \xi^{*}\right\rangle d r \\
&=\pi \int_{0}^{\infty} r\left\langle F^{\prime \prime}(S) \xi \widetilde{\zeta}_{11}, \xi^{*}\right\rangle d r+\pi \int_{0}^{\infty} r\left\langle\boldsymbol{g}_{1}^{\prime}(S) \xi, \xi^{*}\right\rangle d r \\
&=\left\langle F^{\prime \prime}(S) \boldsymbol{\xi}_{2} \boldsymbol{\zeta}_{11}, \xi_{2}^{*}\right\rangle_{L^{2}}+\left\langle\boldsymbol{g}_{1}^{\prime}(S) \boldsymbol{\xi}_{2}, \xi_{2}^{*}\right\rangle_{L^{2}} \\
& \equiv \pi N_{3}^{\prime}
\end{aligned}
$$

and

and 


$$
\begin{aligned}
\left\langle F^{\prime \prime}(S) \boldsymbol{\xi}_{1} \boldsymbol{\zeta}_{12}, \boldsymbol{\xi}_{1}^{*}\right\rangle_{L^{2}}+\left\langle\boldsymbol{g}_{2}^{\prime}(S) \boldsymbol{\xi}_{1}, \boldsymbol{\xi}_{1}^{*}\right\rangle_{L^{2}} & \\
= & \int_{0}^{2 \pi} \cos ^{2} 2 \theta d \theta \int_{0}^{\infty} r\left\langle F^{\prime \prime}(S) \xi \boldsymbol{\zeta}_{12}, \xi^{*}\right\rangle d r \\
& +\int_{0}^{2 \pi} \cos ^{2} 2 \theta d \theta \int_{0}^{\infty} r\left\langle\boldsymbol{g}_{2}^{\prime}(S) \xi, \xi^{*}\right\rangle d r \\
= & \pi \int_{0}^{\infty} r\left\langle F^{\prime \prime}(S) \xi \widetilde{\zeta}_{12}, \xi^{*}\right\rangle d r+\pi \int_{0}^{\infty} r\left\langle\boldsymbol{g}_{2}^{\prime}(S) \xi, \xi^{*}\right\rangle d r \\
= & \left\langle F^{\prime \prime}(S) \boldsymbol{\xi}_{2} \boldsymbol{\zeta}_{12}, \boldsymbol{\xi}_{2}^{*}\right\rangle_{L^{2}}+\left\langle\boldsymbol{g}_{2}^{\prime}(S) \boldsymbol{\xi}_{2}, \boldsymbol{\xi}_{2}^{*}\right\rangle_{L^{2}} \\
& \equiv \pi N_{3}^{\prime \prime} .
\end{aligned}
$$

Here we take the inner product with $\xi_{i}^{*}(i=1,2)$ and obtain $N_{3}=\eta_{1} N_{3}^{\prime}+\eta_{2} N_{3}^{\prime \prime}$. The first term of $N_{1}$ is given as

$$
\begin{aligned}
\frac{1}{6}\left\langle F^{\prime \prime \prime}(S) \xi_{1}^{3}, \boldsymbol{\xi}_{1}^{*}\right\rangle_{L^{2}} & =\frac{1}{6} \int_{0}^{2 \pi} \cos ^{4} 2 \theta d \theta \int_{0}^{\infty} r\left\langle F^{\prime \prime \prime}(S) \xi^{3}, \xi^{*}\right\rangle d r \\
& =\frac{\pi}{8} \int_{0}^{\infty} r\left\langle F^{\prime \prime \prime}(S) \xi^{3}, \xi^{*}\right\rangle d r .
\end{aligned}
$$

Moreover, we have

$$
\begin{aligned}
\frac{1}{6}\left\langle F^{\prime \prime \prime}(S) \xi_{1}^{3}, \boldsymbol{\xi}_{1}^{*}\right\rangle_{L^{2}} & =\frac{1}{2}\left\langle F^{\prime \prime \prime}(S) \boldsymbol{\xi}_{1} \boldsymbol{\xi}_{2}^{2}, \boldsymbol{\xi}_{1}^{*}\right\rangle_{L^{2}}=\frac{1}{6}\left\langle F^{\prime \prime \prime}(S) \boldsymbol{\xi}_{2}^{3}, \boldsymbol{\xi}_{2}^{*}\right\rangle_{L^{2}} \\
& =\frac{1}{2}\left\langle F^{\prime \prime \prime}(S) \boldsymbol{\xi}_{1}^{2} \boldsymbol{\xi}_{2}, \boldsymbol{\xi}_{2}^{*}\right\rangle_{L^{2}} \equiv \pi N_{1}^{\prime}
\end{aligned}
$$

We show the second term of $N_{1}$ as

$$
\begin{aligned}
\left\langle F^{\prime \prime}(S) \boldsymbol{\xi}_{1} \boldsymbol{\zeta}_{4}, \boldsymbol{\xi}_{1}^{*}\right\rangle_{L^{2}} & =\int_{0}^{2 \pi} \cos ^{4} 2 \theta d \theta \int_{0}^{\infty} r\left\langle F^{\prime \prime}(S) \xi \widetilde{\zeta}_{4}, \xi^{*}\right\rangle d r \\
& =\frac{3 \pi}{4} \int_{0}^{\infty} r\left\langle F^{\prime \prime}(S) \xi \widetilde{\zeta}_{4}, \xi^{*}\right\rangle d r .
\end{aligned}
$$

By similar calculations to the above, we have

$$
\begin{aligned}
\left\langle F^{\prime \prime}(S) \boldsymbol{\xi}_{1} \boldsymbol{\zeta}_{4}, \boldsymbol{\xi}_{1}^{*}\right\rangle_{L^{2}}= & \left\langle F^{\prime \prime}(S) \boldsymbol{\xi}_{1} \boldsymbol{\zeta}_{5}, \boldsymbol{\xi}_{1}^{*}\right\rangle_{L^{2}}+\left\langle F^{\prime \prime}(S) \boldsymbol{\xi}_{2} \boldsymbol{\zeta}_{6}, \boldsymbol{\xi}_{1}^{*}\right\rangle_{L^{2}} \\
= & \left\langle F^{\prime \prime}(S) \boldsymbol{\xi}_{2} \boldsymbol{\zeta}_{5}, \boldsymbol{\xi}_{2}^{*}\right\rangle_{L^{2}}=\left\langle F^{\prime \prime}(S) \boldsymbol{\xi}_{1} \boldsymbol{\zeta}_{6}, \boldsymbol{\xi}_{2}^{*}\right\rangle_{L^{2}} \\
& +\left\langle F^{\prime \prime}(S) \boldsymbol{\xi}_{2} \boldsymbol{\zeta}_{4}, \boldsymbol{\xi}_{2}^{*}\right\rangle_{L^{2}} \equiv \pi N_{1}^{\prime \prime} .
\end{aligned}
$$

In this way, we obtain $N_{1}=N_{1}^{\prime}+N_{1}^{\prime \prime}$.

Last, we shall consider the first three terms of $N_{2}$ as

$$
\begin{aligned}
\left\langle\frac{1}{2} F^{\prime \prime \prime}(S) \boldsymbol{\psi}_{1}^{2} \xi_{1}+F^{\prime \prime}(S) \boldsymbol{\psi}_{1} \zeta_{7}+F^{\prime \prime}(S) \xi_{1} \zeta_{1}, \boldsymbol{\xi}_{1}^{*}\right\rangle_{L^{2}} \\
=\frac{1}{2} \int_{0}^{2 \pi} \cos ^{2} \theta \cos ^{2} 2 \theta d \theta \int_{0}^{\infty} r\left\langle F^{\prime \prime \prime}(S) \psi^{2} \xi, \xi^{*}\right\rangle d r+\int_{0}^{2 \pi} \cos ^{2} \theta \cos ^{2} 2 \theta d \theta \int_{0}^{\infty} r\left\langle F^{\prime \prime}(S) \widetilde{\zeta}_{7} \psi, \xi^{*}\right\rangle d r \\
\quad+\int_{0}^{2 \pi} \sin \theta \cos \theta \sin 2 \theta \cos 2 \theta d \theta \int_{0}^{\infty} r\left\langle F^{\prime \prime}(S) \widetilde{\zeta}_{8} \psi, \xi^{*}\right\rangle d r+\int_{0}^{2 \pi} \cos ^{2} \theta \cos ^{2} 2 \theta d \theta \int_{0}^{\infty} r\left\langle F^{\prime \prime}(S) \tilde{\zeta}_{1} \xi, \xi^{*}\right\rangle d r \\
\quad+\int_{0}^{2 \pi} \sin ^{2} \theta \cos ^{2} 2 \theta d \theta \int_{0}^{\infty} r\left\langle F^{\prime \prime}(S) \tilde{\zeta}_{2} \xi, \xi^{*}\right\rangle d r \\
=\frac{\pi}{4} \int_{0}^{\infty} r\left\langle F^{\prime \prime \prime}(S) \psi^{2} \xi, \xi^{*}\right\rangle d r+\frac{\pi}{2} \int_{0}^{\infty} r\left\langle F^{\prime \prime}(S) \tilde{\zeta}_{7} \psi, \xi^{*}\right\rangle d r+\frac{\pi}{2} \int_{0}^{\infty} r\left\langle F^{\prime \prime}(S)\left(\widetilde{\zeta}_{1}+\tilde{\zeta}_{2}\right) \xi, \xi^{*}\right\rangle d r .
\end{aligned}
$$

Similarly, we have

$$
\begin{aligned}
\left\langle\frac{1}{2} F^{\prime \prime \prime}(S) \boldsymbol{\psi}_{1}^{2} \boldsymbol{\xi}_{1}+F^{\prime \prime}(S) \boldsymbol{\psi}_{1} \boldsymbol{\zeta}_{7}+F^{\prime \prime}(S) \boldsymbol{\xi}_{1} \zeta_{1}, \boldsymbol{\xi}_{1}^{*}\right\rangle_{L^{2}} \\
=\left\langle\frac{1}{2} F^{\prime \prime \prime}(S) \boldsymbol{\psi}_{1}^{2} \boldsymbol{\xi}_{2}+F^{\prime \prime}(S) \boldsymbol{\psi}_{1} \boldsymbol{\zeta}_{9}+F^{\prime \prime}(S) \boldsymbol{\xi}_{2} \boldsymbol{\zeta}_{1}, \boldsymbol{\xi}_{2}^{*}\right\rangle_{L^{2}} \\
=\left\langle\frac{1}{2} F^{\prime \prime \prime}(S) \boldsymbol{\psi}_{2}^{2} \boldsymbol{\xi}_{2}+F^{\prime \prime}(S) \boldsymbol{\psi}_{2} \boldsymbol{\zeta}_{8}+F^{\prime \prime}(S) \boldsymbol{\xi}_{2} \boldsymbol{\zeta}_{2}, \boldsymbol{\xi}_{2}^{*}\right\rangle_{L^{2}} \\
=\left\langle\frac{1}{2} F^{\prime \prime \prime}(S) \boldsymbol{\psi}_{2}^{2} \boldsymbol{\xi}_{1}+F^{\prime \prime}(S) \boldsymbol{\psi}_{2} \boldsymbol{\zeta}_{10}+F^{\prime \prime}(S) \boldsymbol{\xi}_{1} \zeta_{2}, \boldsymbol{\xi}_{1}^{*}\right\rangle_{L^{2}} \\
\equiv \pi N_{2}^{\prime} .
\end{aligned}
$$

The last two terms of $N_{2}$ are obtained as 


$$
\begin{aligned}
\left\langle\boldsymbol{\zeta}_{7 x_{1}}, \boldsymbol{\xi}_{1}^{*}\right\rangle_{L^{2}}= & \int_{0}^{2 \pi} \cos ^{2} \theta \cos ^{2} 2 \theta d \theta \int_{0}^{\infty} r\left\langle\widetilde{\zeta}_{7 r}, \xi^{*}\right\rangle d r+\int_{0}^{2 \pi} \sin \theta \cos \theta \sin 2 \theta \cos 2 \theta d \theta \int_{0}^{\infty} r\left\langle\widetilde{\zeta}_{8 r}, \xi^{*}\right\rangle d r \\
& +\int_{0}^{2 \pi} \sin ^{2} \theta \cos ^{2} 2 \theta d \theta \int_{0}^{\infty}\left\langle\widetilde{\zeta}_{7}, \xi^{*}\right\rangle d r+2 \int_{0}^{2 \pi} \sin \theta \cos \theta \sin 2 \theta \cos 2 \theta d \theta \int_{0}^{\infty}\left\langle\widetilde{\zeta}_{7}, \xi^{*}\right\rangle d r \\
& -\int_{0}^{2 \pi} \sin \theta \cos \theta \sin 2 \theta \cos 2 \theta d \theta \int_{0}^{\infty}\left\langle\widetilde{\zeta}_{8}, \xi^{*}\right\rangle d r-2 \int_{0}^{2 \pi} \sin ^{2} \theta \cos ^{2} 2 \theta d \theta \int_{0}^{\infty}\left\langle\widetilde{\zeta}_{8}, \xi^{*}\right\rangle d r \\
= & \frac{\pi}{2} \int_{0}^{\infty} r\left\langle\widetilde{\zeta}_{7 r}, \xi^{*}\right\rangle d r+\frac{\pi}{2} \int_{0}^{\infty}\left\langle\widetilde{\zeta}_{7}, \xi^{*}\right\rangle d r-\pi \int_{0}^{\infty}\left\langle\widetilde{\zeta}_{8}, \xi^{*}\right\rangle d r
\end{aligned}
$$

and

$$
\begin{aligned}
& \left\langle\boldsymbol{\psi}_{1 x_{1}}, \boldsymbol{\xi}_{1}^{*}\right\rangle_{L^{2}}=\int_{0}^{2 \pi} \cos ^{2} \theta \cos 2 \theta d \theta \int_{0}^{\infty} r\left\langle\psi_{r}, \xi^{*}\right\rangle d r \\
& \quad+\int_{0}^{2 \pi} \sin ^{2} \theta \cos 2 \theta d \theta \int_{0}^{\infty}\left\langle\psi, \xi^{*}\right\rangle d r \\
& =\frac{\pi}{2} \int_{0}^{\infty} r\left\langle\psi_{r}-\frac{\psi}{r}, \xi^{*}\right\rangle d r .
\end{aligned}
$$

Similarly as before, we have

$$
\left\langle\boldsymbol{\zeta}_{7 x_{1}}, \boldsymbol{\xi}_{1}^{*}\right\rangle_{L^{2}}=\left\langle\boldsymbol{\zeta}_{9 x_{1}}, \boldsymbol{\xi}_{2}^{*}\right\rangle_{L^{2}}=\left\langle\boldsymbol{\zeta}_{8 x_{2}}, \boldsymbol{\xi}_{2}^{*}\right\rangle_{L^{2}}=\left\langle\boldsymbol{\zeta}_{10 x_{2}}, \boldsymbol{\xi}_{1}^{*}\right\rangle_{L^{2}} \equiv \pi N_{2}^{\prime \prime}
$$

and

$$
\begin{aligned}
\left\langle\boldsymbol{\psi}_{1 x_{1}}, \boldsymbol{\xi}_{1}^{*}\right\rangle_{L^{2}} & =\left\langle\boldsymbol{\psi}_{1 x_{2}}, \boldsymbol{\xi}_{2}^{*}\right\rangle_{L^{2}}=\left\langle\boldsymbol{\psi}_{2 x_{1}}, \boldsymbol{\xi}_{2}^{*}\right\rangle_{L^{2}}=-\left\langle\boldsymbol{\psi}_{2 x_{2}}, \boldsymbol{\xi}_{1}^{*}\right\rangle_{L^{2}} \\
& \equiv \pi N_{2}^{\prime \prime \prime}
\end{aligned}
$$

Therefore, we arrive at $N_{2}=N_{2}^{\prime}+N_{2}^{\prime \prime}-\beta^{\prime} N_{2}^{\prime \prime \prime}$.
[1] V. K. Vanag and I. R. Epstein, Chaos 17, 037110 (2007).

[2] K. Krisher and A. Mikhailov, Phys. Rev. Lett. 73, 3165 (1994).

[3] M. Or-Guil, M. Bode, C. P. Schenk, and H.-G. Purwins, Phys. Rev. E 57, 6432 (1998).

[4] Y. Nishiura, T. Teramoto, and K.-I. Ueda, Chaos 15, 047509 (2005).

[5] T. Ohta, Physica D 151, 61 (2001).

[6] S. V. Gurevich, H. U. Bödeker, A. S. Moskalenko, A. W. Liehr, and H.-G. Purwins, Physica D 199, 115 (2004).

[7] L. M. Pismen, Phys. Rev. E 74, 041605 (2006).

[8] Y. Sumino, N. Magome, T. Hamada, and K. Yoshikawa, Phys. Rev. Lett. 94, 068301 (2005).

[9] U. Thiele and E. Knobloch, Phys. Rev. Lett. 97, 204501 (2006).
[10] X. Jiang, D. A. Bruzewicz, A. P. Wong, M. Piel, and G. M. Whitesides, Proc. Natl. Acad. Sci. U.S.A. 102, 975 (2005).

[11] Y. T. Maeda, J. Inose, M. Y. Matsuo, S. Iwaya, and M. Sano, PLoS ONE 3, e3734 (2008).

[12] S.-I. Ei, J. Dyn. Differ. Equ. 14, 85 (2002).

[13] S.-I. Ei, M. Mimura, and M. Nagayama, Discrete Contin. Dyn. Syst. 14, 31 (2006).

[14] Y. A. Kuznetsov, Elements of Applied Bifurcation Theory, 2nd ed. (Springer-Verlag, Berlin, 1998).

[15] M. R. E. Proctor and C. A. Jones, J. Fluid Mech. 188, 301 (1988).

[16] D. Armbruster, J. Guckenheimer, and P. Holmes, Physica D 29, 257 (1988).

[17] T. R. Smith, J. Moehlis, and P. Holmes, Physica D 211, 347 (2005). 